\title{
Traditional Chinese Medicine for Essential Hypertension: A Clinical Evidence Map
}

\author{
Yan Zhang $\mathbb{D}^{1,2,3}$ Biqing Wang, ${ }^{4,2,3}$ Chunxiao Ju, ${ }^{4,2,3}$ Lu Liu, ${ }^{4,2,3}$ Ying Zhu, ${ }^{4,2,3}$ Jun Mei, ${ }^{2,3}$ \\ Yue Liu $\mathbb{D}^{1,3}$ and Fengqin $\mathrm{Xu} \mathbb{1}^{2,3}$ \\ ${ }^{1}$ Graduate School of China Academy of Chinese Medical Sciences, Beijing 100700, China \\ ${ }^{2}$ Center of Geriatrics Diseases, Xiyuan Hospital, China Academy of Chinese Medical Sciences, Beijing 100091, China \\ ${ }^{3}$ Cardiovascular Disease Team, China Center for Evidence-Based Medicine of TCM, Beijing 100091, China \\ ${ }^{4}$ Graduate School of Beijing University of Chinese Medicine, Beijing 100029, China \\ ${ }^{5}$ Center of Cardiovascular Diseases, Xiyuan Hospital, China Academy of Chinese Medical Sciences, Beijing 100091, China
}

Correspondence should be addressed to Yue Liu; liuyueheart@hotmail.com and Fengqin Xu; dr.xufengqin@outlook.com

Received 7 June 2020; Revised 14 August 2020; Accepted 11 November 2020; Published 19 December 2020

Academic Editor: Hong Chang

Copyright ( 92020 Yan Zhang et al. This is an open access article distributed under the Creative Commons Attribution License, which permits unrestricted use, distribution, and reproduction in any medium, provided the original work is properly cited.

\begin{abstract}
We systematically retrieved and summarised clinical studies on traditional Chinese medicine (TCM) for the prevention and treatment of essential hypertension (EH) using the evidence map. We aimed to explore the evidence distribution, identify gaps in evidence, and inform on future research priorities. Clinical studies, systematic reviews, guidelines, and pathway studies related to TCM for the prevention and treatment of EH, published between January 2000 and December 2019, were included from databases CNKI, WanFang Data, VIP, PubMed, Embase, and Web of Science. The distribution of evidence was analysed using text descriptions, tables, and graphs. A total of 9,403 articles were included, including 5,920 randomised controlled studies (RCTs), 16 guidelines, expert consensus and path studies, and 139 systematic reviews (SRs). The articles publishing trend increased over time. This study showed that the intervention time of TCM was concentrated at 4-8 weeks, mainly through Chinese herbal medicine (CHM) for the prevention and treatment of elderly hypertension and the complications. A Measurement Tool to Assess Systematic Reviews (AMSTAR) scores of the included reviews ranged from 2 to 10. Most of the SRs had a potentially positive effect $(n=120)$, mainly in 5-8 score. Primary studies and SRs show potential benefits of TCM in lowering blood pressure, lowering the TCM syndrome and symptom differentiation scores (TCM-SSD scores), improving the total effective rate, and reducing the adverse events. The adjunctive effect of TCM on improving the total effective rate, lowering the blood pressure, lowering the TCMSSD scores, and lowering the adverse effects was only supported by low-quality evidence in this research. The evidence map was used to show the overall research on TCM for the treatment of EH; however, due to the existing problems of the primary studies, the current research conclusion needs further research with higher quality and standardisation.
\end{abstract}

\section{Introduction}

Hypertension has become a primary global disease and is an important global public health challenge [1]. According to literature, in 2000, 26.4\% of adults worldwide suffered from high blood pressure. It is estimated that by $2025,29.2 \%$ of people in the world will suffer from high blood pressure [2]. There is currently an upward trend of the hypertension prevalence and mortality rates among Chinese residents and it is predicted that by 2030, the annual economic burden of cardiovascular disease deaths caused by hypertension in China will reach \$6-9 million [3]. A prospective epidemiological study of 47,000 residents in 115 urban and rural communities in China showed that the prevalence rate, awareness rate, treatment rate, and control rate of hypertension in China were $41.9 \%, 41.6 \%, 34.4 \%$, and $8.2 \%$, respectively, indicating that prevention, detection, treatment, and control of hypertension should be prioritised [4].

Antihypertensive therapy is currently widely used; however, its understanding, management, and control are 
not well known due to the adverse effects and intolerance of antihypertensive drugs that the patients currently face [5]. Therefore, more attention must be given to complementary and alternative medical treatments. Systematic reviews (SRs) have shown that traditional Chinese medicine (TCM) has a significant effect on lowering blood pressure but there is little research on its underlying intervention mechanisms $[6,7]$.

As an evidence integration method, evidence mapping can integrate evidence of various study types under a research topic and comprehensively demonstrate the problems in the research topic, thereby depicting a complete picture of the research field [8-10]. Several evidence mapping reports have been published on the Chinese medical fields such as acupuncture, Tai Chi, massage, and angelica; however, they only included randomised controlled studies (RCTs) and SRs [11-14]. However, the clinical evidence for the prevention and treatment of hypertension by TCM is unclear. Therefore, this study used an evidence map to systematically find relevant literature (observational studies, RCTs, SRs, guidelines, and expert consensus) on the clinical prevention and treatment of essential hypertension, in order to better understand the distribution of evidence in this field, identify gaps in evidence, and provide potential information for priority areas.

\section{Methods}

2.1. Database and Search Strategies. The literature searches were conducted using PubMed, Web of Science, Embase, Chinese National Knowledge Infrastructure (CNKI), Chinese Scientific Journal Database (VIP), and WanFang data. The search was restricted from January 1, 2000, to December 31, 2019. We searched the Chinese database using "hypertension". The retrieval subjects are limited to TCM, integrated Chinese and Western medicines, TCM internal medicine, surgery of Chinese medicine, gynaecology of Chinese medicine, paediatrics of Chinese medicine, and other TCM-related subjects. English database retrieval was divided into two parts. The search terms for the first retrieval included: ("hypertension" OR "blood pressure, high" OR "blood pressures, high" OR "high blood pressure" OR "high blood pressures") AND ("medicine, Chinese traditional" OR "traditional Chinese medicine" OR "traditional medicine, Chinese" OR "Chinese medicine, traditional" OR "herbal medicine" OR "drugs, Chinese herbal" OR "herbal formula" OR "Chinese herbal medicine" OR "Chinese herb therapy" OR "Chinese herb" OR "herb therapy" OR "herbal remedy" OR "acupuncture"). The second retrieval search term was "hypertension" + hypertension-related formulas and nondrug therapy that frequently appeared in the meta-analysis in the Chinese database; the two retrievals were combined. The literature searched included academic journals, graduation theses, and conference papers.

\subsection{Inclusion Criteria. The inclusion criteria were as follows:}

(1) Type of study: RCTs, nonrandomised controlled trials (non-RCTs), cohort studies, case-control studies, cross-sectional studies, real-world studies (RWS), systematic reviews, meta-analyses, expert consensus, guidelines, and clinical pathway studies on TCM intervention for hypertension

(2) Type of participants: the patients that met the diagnostic criteria of essential hypertension. There was no limitation on the age, sex, race, time of onset, and cases of the source

(3) Type of intervention: TCM (Chinese herbal medicine (CHM) (decoction, tablet, pill, powder, granule, capsule, oral liquid, or injection), nondrug therapy (acupuncture, qigong, massage, and Baduanjin, etc.)), nursing of TCM, or above measures combined with conventional Western medicine that was used in the treatment groups. The comparison interventions were conventional Western medicine, placebo, or blank controls

(4) Type of outcome: the main outcomes included blood pressure (BP), total effective rate, TCM syndrome and symptom differentiation (TCM-SSD) scores, and adverse events. TCM prevention and treatment, TCM syndrome type, and duration of TCM intervention

2.3. Exclusion Criteria. (1) Clinical experience, (2) clinical trial protocols, (3) meeting abstracts, (4) no full-text, (5) redundant publication, and (6) fundamental researches were excluded.

2.4. Literature Screening and Data Extraction. Four authors independently conducted the literature search, study selection, and data extraction, and 2 authors conducted it as a group. The extracted data included the following: (1) basic information: author, publication year, study object and disease, intervention measures, total sample size, and outcome indicators; (2) study type ((i) intervention study: RCTs, non-RCTs, (ii) observational study: a cohort study, casecontrol study, and cross-sectional study, (iii) secondary study: SRs, guidelines, and clinical pathway studies, (iv) RWS); (3) treatment categories (CPM, CHM, nursing of TCM, acupuncture, massage, TCM exercise therapy, auricular point, acupoint application, multimethod combination, and others); (4) complicating diseases (cerebral haemorrhage, cerebral infarction, angina pectoris/myocardial ischaemia, arrhythmia, diabetes/abnormal glucose metabolism, cardiac insufficiency, anxiety and depression, renal diseases, eye diseases, insomnia/sleep disorders, hyperlipidaemia, hyperuricaemia, metabolic syndrome, atherosclerosis, etc.); and (5) the duration of therapeutic intervention. Disagreements were resolved by discussion, and a consensus was reached through a third party (J. Mei).

2.5. Quality Assessment of the Included Systematic Reviews. A Measurement Tool to Assess SRs (AMSTAR), which consists of 11 items, was used to evaluate the methodological quality of all the included SRs. For each item, "Yes," "No," 
"Can't answer," or "Not applicable" was assigned according to judgement criteria of AMSTAR. The number of "yes" was counted as the total AMSTAR score. A score of 4 or less was considered low quality, a score of 5 to 8 was medium quality, and a score of 9 or more was high quality $[15,16]$. Based on the SRs' clinical effectiveness, it was further divided into 4 categories: "evidence of no effect," "unclear evidence," "evidence of a potentially positive effect," and "evidence of a positive effect" [13]. The category "evidence of no effect" meant that the effect of the control group is equal to or better than that of the TCM observation group. "Unclear evidence" meant that the result of a systematic review of similar contents is controversial, or the evidence is summarised as inconclusive by the original study's author. "Evidence of a potentially positive effect" referred to the systematic review of all included clinical studies, combined results, and statistical evidence to show effectiveness but the lack of basic and auxiliary evidence made it difficult to produce positive and reliable conclusions. "Evidence of a positive effect" meant that statistics showed that TCM therapy had a significant effect and that the authors of the systematic review had no major doubts regarding the current evidence and recommend the therapy.

2.6. Data Analysis and Presentation. EXCEL 2013 was used to integrate and process the data. The data summary and analysis are shown as text and charts. The distribution of the development trend is depicted as a line chart, the distribution of category proportions as a pie chart, and the distribution of evidence as bubble plots and heatmap.

\section{Results}

3.1. Description of the Included Trials. The initial search retrieved 55,197 articles from the six databases. After removing duplicates, 39,162 trials were identified. After screening the titles and abstracts, 10,302 trials were retained. By browsing the full-text articles, we further excluded 899 records. In the end, 9,403 studies were reviewed, including primary studies $(n=9,243)$, systematic reviews $(n=144)$, and guidelines, expert consensus, and path studies $(n=16)$ (Figure 1).

3.2. Trends in Publication Year of Clinical Studies. A total of 9,403 studies were included from January 2000 to December 2019. The number of studies showed an overall rising trend with a peak in 2018 at home and 2015 abroad, respectively (see Figure 2). The TCM role is increasingly being suspected in the prevention and treatment of hypertension, both in China and worldwide.

3.3. Type and Scale of Clinical Studies. The clinical studies were mainly RCTs, including intervention studies (RCTs $(n=5,920,63.0 \%)$, non-RCTs $(n=2,133,22.7 \%))$, observational studies $(n=1185,12.6 \%)$, RWS $(n=5,0.1 \%)$, and SRs $(n=144,1.5 \%)$. The minimum sample size of the RCT was 10 and the maximum was 2,110 [17]. The maximum sample size of the observational study was 154,083 cases [18], and the sample size of the interventional study was mostly in the range of 60 to 100 cases. In RWS, the sample size ranged from 1,544 to 30,034 cases [19] (see Table 1 ).

3.4. Research on Syndrome and Constitution. A total of 848 clinical studies on TCM syndromes of hypertension were included, of which the syndrome distribution ranked first with a total of 162 ; others included hypertension syndromes and clinical indicators in young and middle-aged people $(n=4)$ [20-23], syndromes in elderly hypertension $(n=124)$, hypertension stages and grades $(n=9)$ [24], four diagnosis information and TCM syndromes $(n=1)$ [25], and TCM syndromes and clinical indicators in grade 3 hypertension $(n=2)[26,27]$. Regarding comorbidity, there were 2 cases of hypertension with arrhythmia, 22 cases of atherosclerosis, 9 cases of a cerebral haemorrhage, 19 cases of cerebral infarction, and 32 cases of diabetes. Studies on the correlation between syndromes and clinical indicators mainly involved indicators such as homocysteine, blood lipid, blood glucose, vascular function, and inflammation. A total of 245 studies on the TCM constitution of hypertension were included, of which there were 12 constitution and syndrome types, mainly involving the phlegm-dampness syndrome [28]. As the syndrome type and constitution articles involved more than 100 kinds of clinical indicators, only the first 36 indicators were shown (see Figure 3).

The bubble plot shows the syndrome and constitution and mainly on a wide range of hypertension and elderly patients with hypertension; however, there are few studies on prehypertension and hypertension grades.

3.5. Categories of TCM Prevention and Treatment. TCM prevention and treatment schemes are mainly divided into 10 categories, including CHM decoction $(n=4,059,49.6 \%)$, Chinese patent medicine $(n=1,916,23.4 \%)$, acupuncture ((electroacupuncture and meridional acupuncture) $(n=505$, $6.2 \%))$, massage $(n=109,1.3 \%)$, auricular point and auricular acupuncture $(n=163,2.0 \%)$, acupoint application $(n=149,1.8 \%)$, TCM exercise therapy (Tai Chi, Baduanjin, wu qinxi) $(n=52,0.6 \%)$, TCM comprehensive nursing ( $n=311,3.8 \%)$, multitherapy $(n=516,6.3 \%)$, and others (pediluvium, fumigation, etc.) $(n=405,4.9 \%)$ ) (Figure 4$)$. $\mathrm{CHM}$ and nondrug therapies are widely used for treating hypertension.

3.6. Clinical Evaluation of the TCM Treatment Schemes. Regarding hypertension and the complications, more than 5 separate evaluations of clinical studies included 14 injections such as compound Danshen injection, astragalus injection, Shengmai injection, and Danhong injection; 12 oral CPM such as the Niuhuang Jiangya pill, Liuwei Dihuang pill, and Songling Xuemaikang capsule; and 13 types of oral CHM decoctions such as Xuefu Zhuyu decoction, Banxia Bbaizhu Tianma decoction, and Lingjiao Gouteng decoction (Figures 5 and 6). The main treatment methods included the calming liver-yang method, resolving phlegm and 


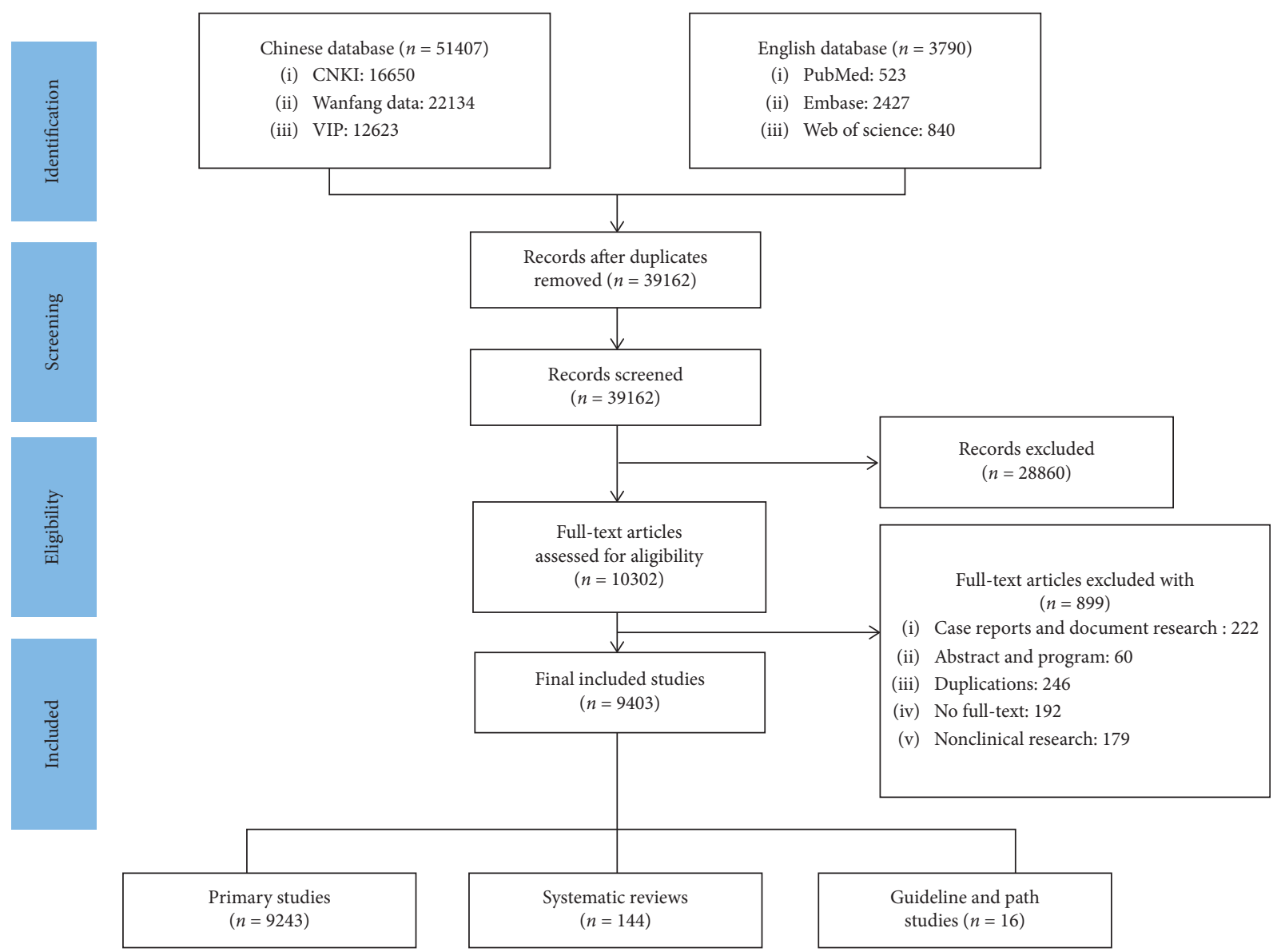

FIgURE 1: Study flow diagram.

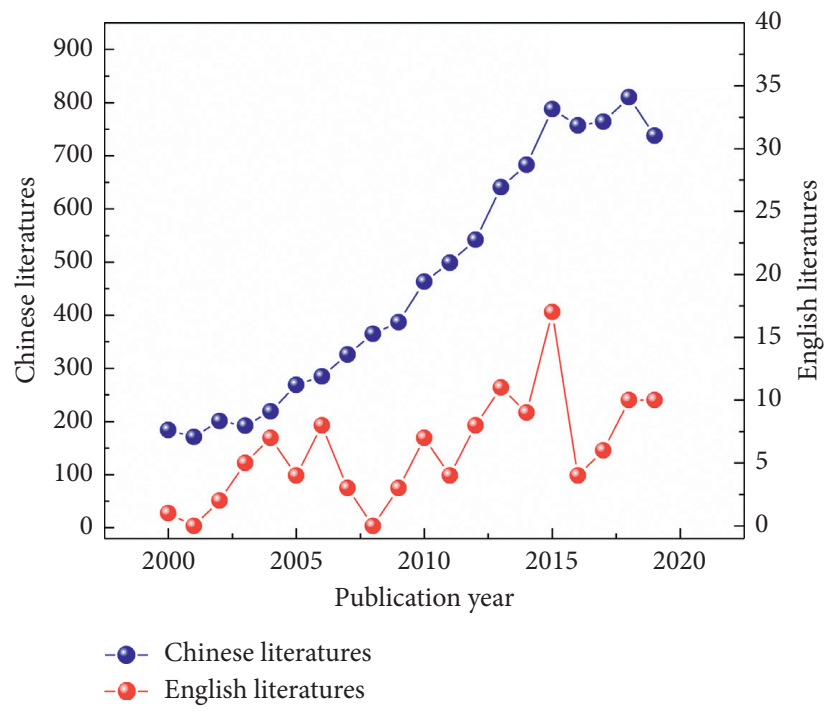

Figure 2: Annual trends in the clinical research literature. The blue line denotes the number of Chinese literature, and the red line denotes the number of English literature.

quenching wind, promoting blood circulation, and removal of blood stasis. The combination modes were mostly CHM decoction in combination, CHM decoction combined with CPM, and integrated Chinese and Western medicines.
The evaluation of TCM prevention and treatment schemes was divided into several types of indicators: total effective rate, $\mathrm{BP}$, TCM-SSD scores, clinical symptoms, blood lipid levels, inflammatory indicators (e.g., C-reactive 
TABLE 1: Clinical study scale.

\begin{tabular}{lccc}
\hline Study sample size $(n)$ & Number of research articles $(n(\%))$ & Real-world study \\
& Observational study & 0 \\
$n<60$ & $1252(15.54)$ & $41(3.46)$ & 0 \\
$60 \leq n<100$ & $3977(49.38)$ & $146(12.32)$ & 0 \\
$100 \leq n<300$ & $2638(32.76)$ & $595(50.21)$ & 0 \\
$300 \leq n<1000$ & $167(2.07)$ & $316(26.67)$ & $5(100.00)$ \\
$n \geq 1000$ & $19(0.24)$ & $87(7.34)$ & 5 \\
Total & 8053 & 1185 & \\
\hline
\end{tabular}

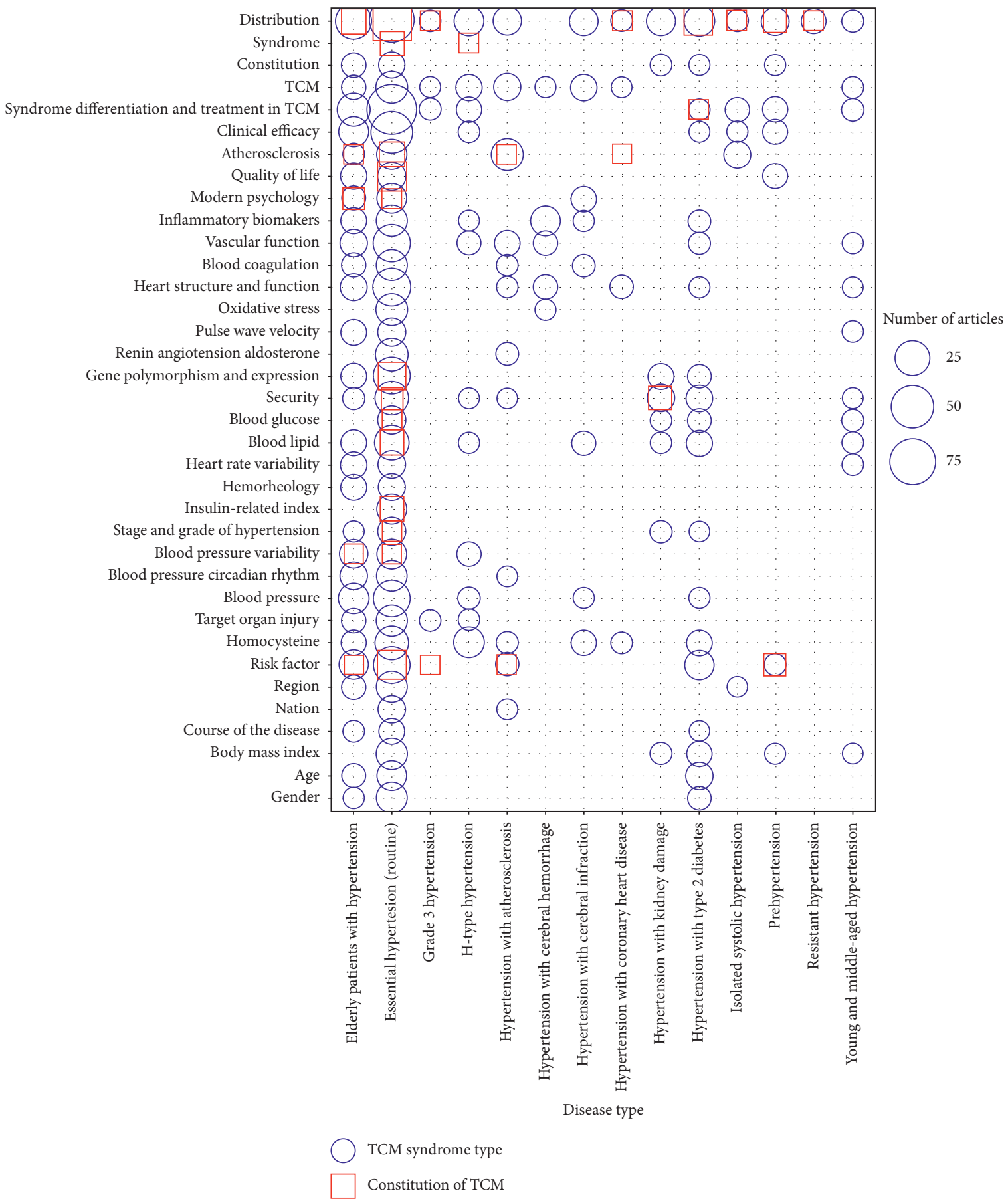

FIGURE 3: Evidence distributions of clinical studies on syndrome and constitution. Objects of study ( $x$-axis) and research content ( $y$-axis). The red square indicates the constitution of TCM and the blue bubbles indicate the TCM syndrome types. 


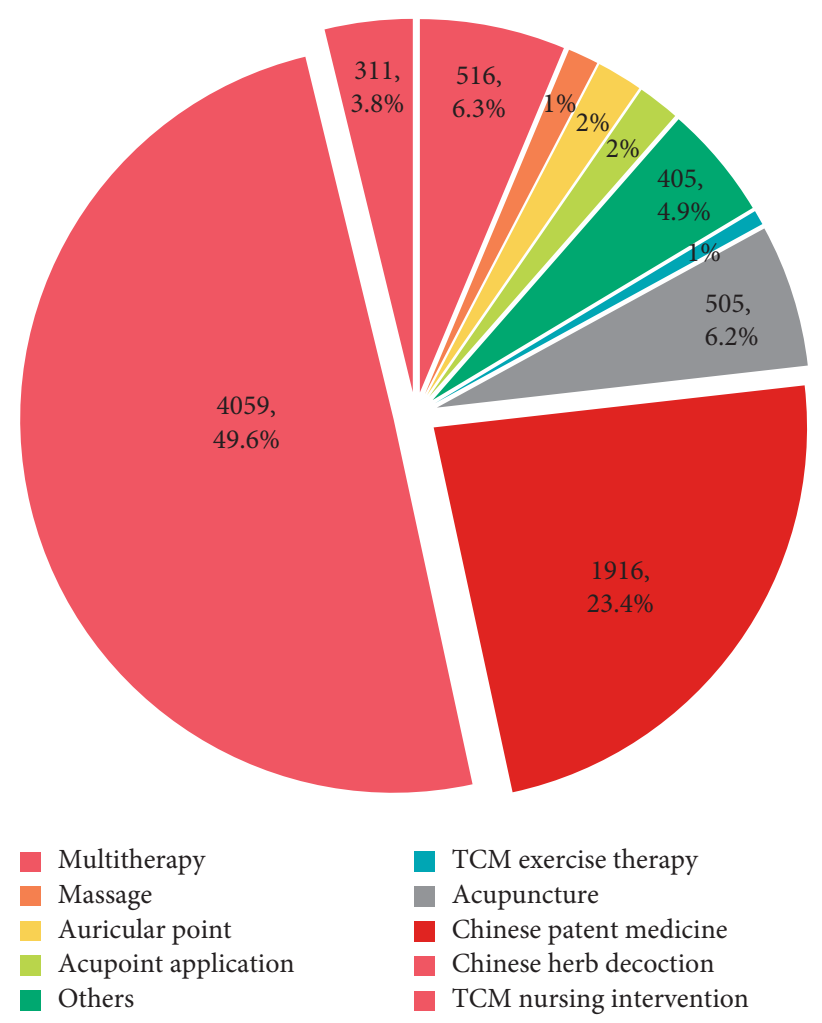

FIGURE 4: Category distribution of the prevention and treatment of hypertension by TCM.

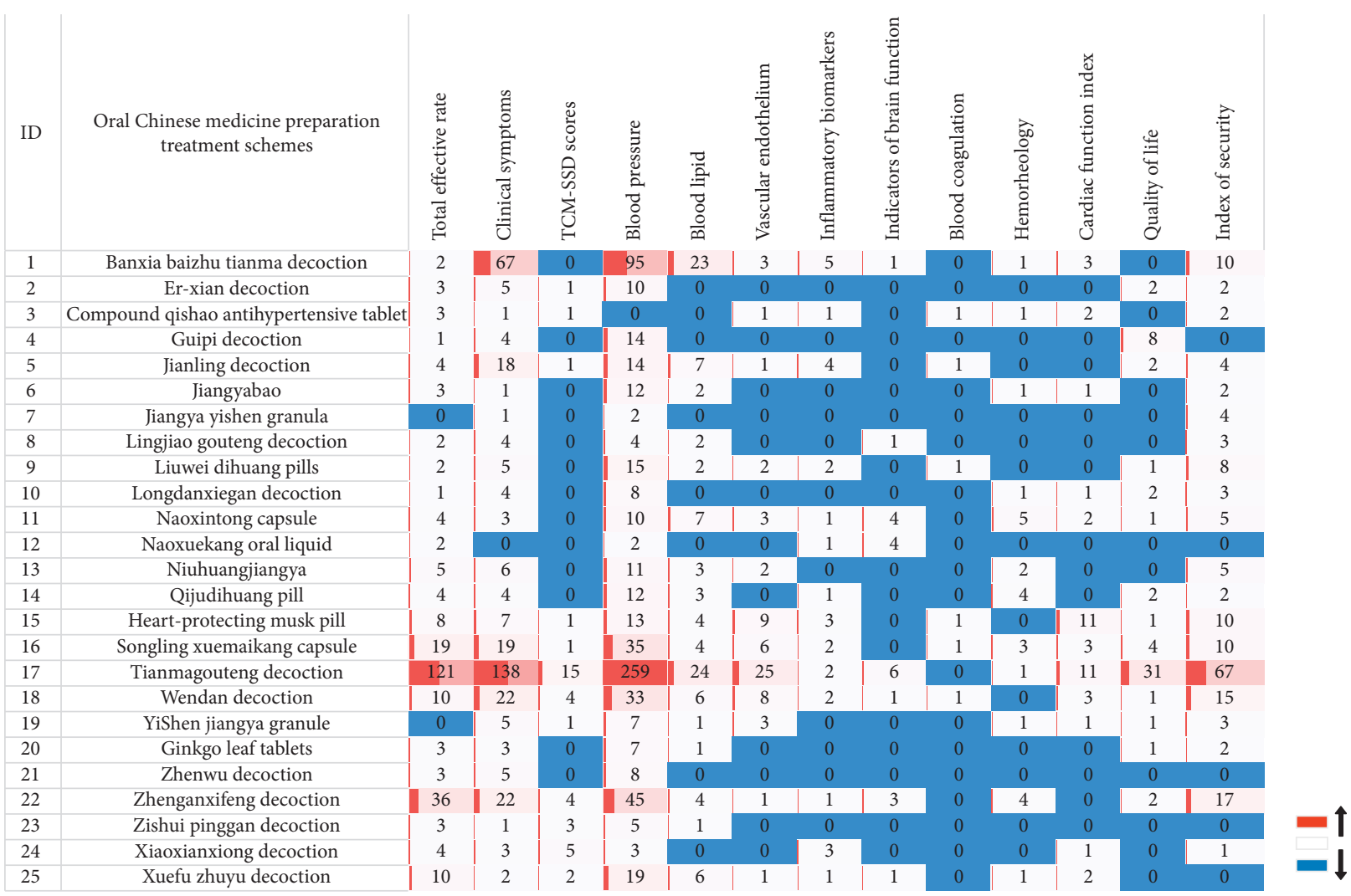

Figure 5: Distribution of clinical evidence for the prevention and treatment of hypertension by oral Chinese herbal preparations. The change of "blue-white-red" colour represents the number of research literature from low to high, and numbers represent the corresponding number of literature. The evaluation index of clinical research is in $x$-axis and oral Chinese medicine preparation is in $y$-axis. 


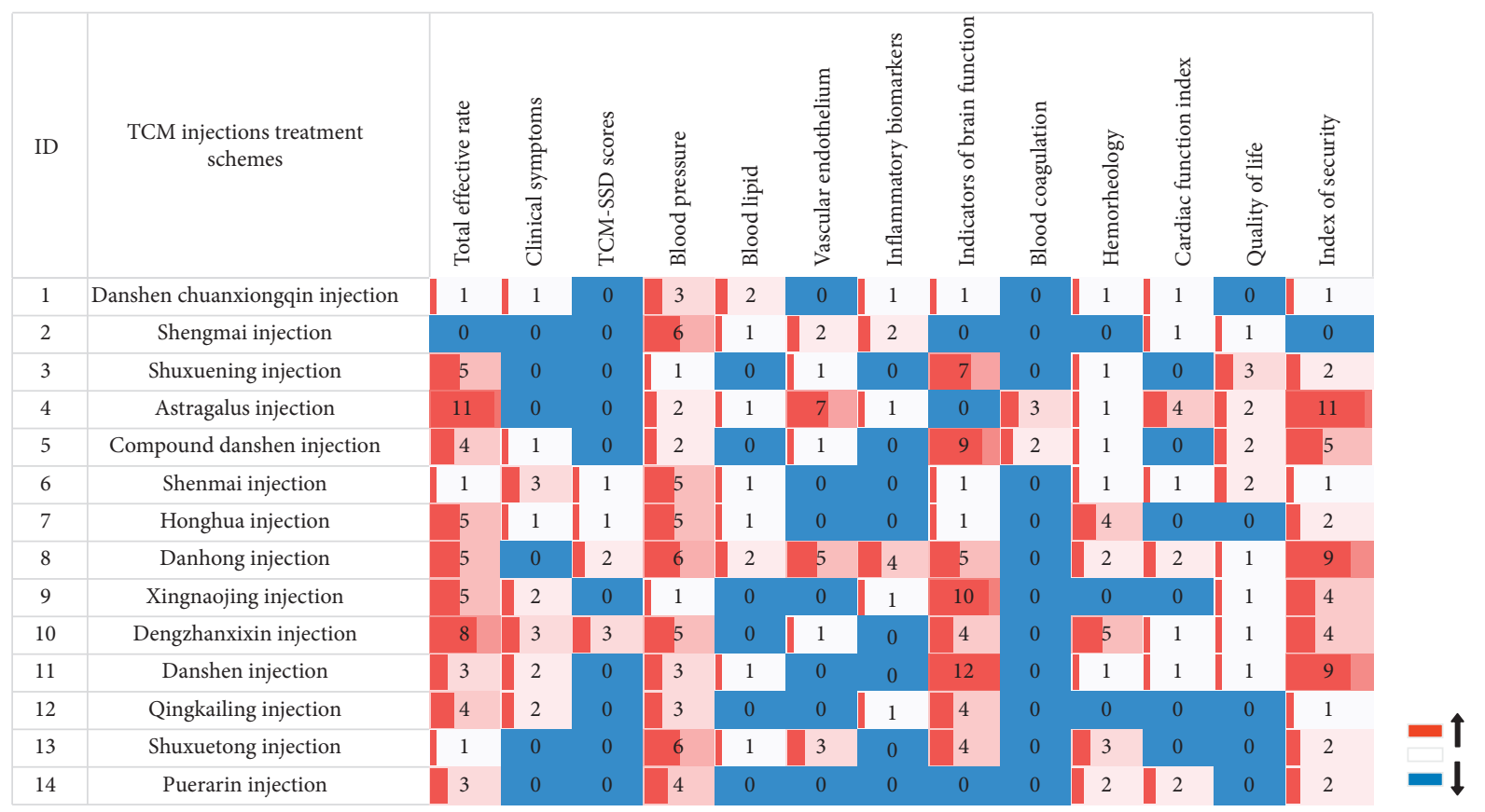

FIGURE 6: Distribution of clinical evidence on the prevention and treatment of hypertension by TCM injections. The change of "blue-whitered" colour represents the number of research literature from low to high and numbers represent the corresponding number of literature. The evaluation index of clinical research is in $x$-axis and traditional Chinese medicine injection is in $y$-axis.

protein, inflammatory factors, etc.), brain function evaluation indicators (e.g., National Institute of Health stroke scale (NIHSS), neurological function score, cerebral haematoma absorption, etc.), cardiac function indicators (such as myocardial injury markers, cardiac structure indicators, cardiac function classification, etc.), hemorheology (e.g., blood viscosity, blood flow velocity, etc.), QOL (e.g., SF-36 quality of life scale, etc.), and safety evaluation (e.g., adverse events, rebleeding event, liver and kidney function). The research evidence distribution of commonly used oral CHM preparations and traditional Chinese medicine injections for the prevention and treatment of hypertension is shown in a heatmap (Figures 5 and 6).

Studies of CHM injection and oral traditional Chinese medicine preparations showed that the evaluation indexes of hypertension were mostly related to complications. The total effective rate, $\mathrm{BP}$, brain function evaluation indicators, and safety index of TCM injection had a high degree of attention, shown in red. Blood coagulation and TCM-SSD scores had low attention, shown in blue. The total effective rate, clinical symptoms, BP, and safety index of oral Chinese medicine preparation were highly relevant in the clinical studies. The indicators of blood coagulation, hemorheology, and brain function received little attention, and the research directions were generally consistent.

\subsection{Investigation of the Application of TCM Prevention and} Treatment Schemes. RWS found that the 30,034 hypertension patients in 16 AAA-grade hospitals were mainly treated with intravenous drugs, among which the 3 traditional Chinese medicine preparations, the Danhong injection, Shuxue Ning injection, and Ginkgo Biloba extract, were used more than $10 \%$ of the total drugs used [19]. The Beijing Hospital found that Liuwei Dihuang pill had the highest comprehensive ranking for the use and frequency of CPM from 2008 to 2010 [29]. Regarding CHM decoction, a cohort study involving 154,083 people in Taiwan from 2003 to 2009 showed that about $80 \%$ of patients used traditional Chinese medicine at least once. Tianma Gouteng decoction and salvia miltiorrhiza were the most frequently used Chinese medicine [18]. From 1996 to 2005, the main herbal medicine types for hypertension in the Beijing area were tonify deficiency medicine, levelling liver and calming wind drugs, heatclearing drugs, blood-activating and stasis-eliminating compound, and damp-clearing drugs [30]. Similarly, the study found that in the past 30 years, the first 5 effective treatments were activating the blood and dissolving stasis, xifeng antispasmodic, benefit qi and blood, smooth liver yang, and removal of pathogenic heat from the blood [31]. In summary, there was a consistent use of medication for hypertension prescriptions.

3.8. TCM for the Treatment of Hypertension and Complications. The current main research target is the middle-aged and elderly hypertension and mainly involves grade 1-2 hypertension. A total of 1,214 studies focussed on elderly hypertension and 42 studies focussed on the treatment of middle-aged and young patients with hypertension. A total of 2,579 studies focussed on hypertension and its complications, accounting for $27.43 \%$ of the total research. The top 3 complications were intracerebral haemorrhage $(n=693,26.9 \%)$, kidney damage $(n=397,15.4 \%)$, and diabetes mellitus/abnormal glucose $(n=378,14.7 \%)$ (Table 2$)$. A total of 1,309 articles commonly used intervention of 
traditional Chinese medicine preparations and 1,170 articles used the analysis intervention duration, most of which were concentrated in a $4-8$ week period ( $n=547,46.8 \%)$, of which only 3 articles of more than 42 months of intervention were present in the strongly exposed group [32-34], suggesting that the research time limit of TCM intervention in hypertension was generally shorter (Figure 7).

3.9. Evidence Quality and Evaluation of the Included Systematic Reviews. A total of 144 systematic reviews were retrieved; there were 5 overviews of SRs without analysis [35-39]. The evidence map for TCM is based on the 139 published systematic reviews, including the Chinese herbal medicine studies $(n=92)$ and nondrug therapy studies $(n=47)$. The single CHM and nondrug therapy were combined into one category, respectively. According to the types of TCM intervention, the intervention principles were divided into 23 types: acupuncture $(n=24)$, qigong $(n=2)$, Tai Chi $(n=2)$, baduanjin $(n=4)$, massage $(n=2)$, auricular point $(n=5)$, acupoint application $(n=3)$, songling xuemai kang capsule $(n=4)$, tongxinluo capsule $(n=2)$, yangxueqingnao granule $(n=3)$, tianmagouteng decoction $(n=11)$, niuhuang jiangya $(n=4)$, banxia baizhu tianma decoction $(n=5)$, buzhong yiqi decoction $(n=2)$, xuefu zhuyu decoction $(n=2)$, Promoting blood circulation and removing blood stasis injection (PBCRBSI) $(n=3)$, pinggan-qianyang treatment $(n=2)$, qiju dihuang pill $(n=2)$, compound qi ma capsule $(n=3)$, tongxinluo capsule $(n=2)$, zhengan xifeng decoction $(n=2)$, tonifying kidney herbs $(n=8)$, CHM $(n=40)$, and nondrug therapy of TCM $(n=4)$. The quality of the included reviews is shown in Figure 8.

According to the AMSTAR scale evaluation, the most qualified item, 9, had 138 SRs. However, 137 SRs did not provide the preliminary design scheme, 106 reviews did not consider the retrieval and inclusion of the grey literature, 70 SRs did not perform a comprehensive literature search, 29 SRs did not properly apply the scientific quality of the included studies to the derivation of conclusions, 31 SRs did not assess and document the scientific quality of the included studies, 138 SRs did not provide the list of included and excluded research literature, 40 SRs did not assess the likelihood of publication bias assessment, and 117 SRs did not provide a conflict of interest statement. One review met 10 criteria [40] and nine reviews met 9 criteria [6, 7, 41-47]. The authors considered these 10 systematic reviews to be of high quality. A total of 94 systematic reviews were of moderate quality and met the 8 AMSTAR criteria $(n=19), 7$ criteria $(n=27), 6$ criteria $(n=27)$, and 5 criteria $(n=21)$. The other 35 systematic reviews were of the lower quality and met 4 criteria $(n=14), 3$ criteria $(n=10)$, or 2 criteria $(n=11)$.

Regarding clinical evidence with SRs, a small number of SRs had unclear evidence $(n=16)$ [40, 42, 43, 45, 47-58]. Most of the SRs had a potentially positive effect $(n=120)$ [7, 44, 46, 59-114], concentrated in 5-8 score. Three SRs were positive, concentrated in the $7-9$ score. $[6,115,116]$. To summarise, most of the included SRs were based on the poor quality of primary studies and the quality of clinical efficacy of most primary outcomes was a potentially positive effect (86\%).

\subsection{A General Overview of the Systematic Reviews}

3.10.1. CHM plus Antihypertensive Drugs versus Antihypertensive Drugs. In the 139 SRs, most of the intervention measures were CHM combined with Western medicine (77, $55.4 \%$ ). Forty-three SRs (quality range $=2-8$ ) included SBP as an outcome measure, 38 SRs (quality range $=2-9$ ) included DBP as an outcome measure, 20 SRs (quality range $=2-8$ ) included total effective rate as an outcome measure, and 18 SRs (quality range $=4-9$ ) included TCMSSD scores as an outcome measure. There are significant differences in the effect of CHM plus antihypertensive drugs for lowering SBP $(n=35,81.4 \%)[6,41,43,45,46,58,61,62$, $64,65,67-69,78,80,92,93,95,96,99,101,102,114$, 117-127], lowering DBP $(n=26, \quad 68.4 \%) \quad[6$, $41,45,46,58,61,62,64,68,69,78,80,93,95,101,102,119$, 121-126], improving total effective rate $(n=18,90.0 \%)$ $[58,76,82,83,99,103,107,108,111,112,114,121,122,126$, $128-131]$, and lowering TCM-SSD scores $(n=17,94.4 \%)$ $[7,41,46,48,62,76,79,94,95,99,102,112,130,132-135]$ than the antihypertensive drugs. The Xinmaitong (6 RCTs; quality $=7$ ) and songling xuemakang capsules (4 RCTs; quality $=8)$, combined with antihypertensive drugs, significantly lowered the SDP and DBP and improved clinical efficacy, with low heterogeneity. Clinical evidence was the recommended level $[115,116]$.

3.10.2. CHM versus Antihypertensive Drugs. Among the 139 SRs, 42 SRs (27.3\%) were of the CHM therapy alone. The outcome measures SBP, DBP, total effective rate, and TCMSSD scores included 21 SRs, 20 SRs, 13 SRs, and 10 SRs, respectively. There were significant differences in the effect of CHM for lowering SBP $(n=12, \quad 57.1 \%)$ $[7,45,58,62,92,93,102,116,121,127,136,137]$, lowering DBP $(n=7,35.0 \%)[7,45,62,93,102,116,137]$, improving the total effective rate $(n=6, \quad 46.1 \%)$ $[55,81,110,130,138,139]$, and lowering the TCM-SSD scores $(n=9,90.0 \%)[7,48,62,84,94,102,112,115,133]$.

3.10.3. Nondrug Therapy plus Antihypertensive Drugs versus Antihypertensive Drugs. Of the 139 SRs, 38 SRs (27.3\%) were nondrug therapy combined with Western medicine. The outcome measures of SBP, DBP, total effective rate, and TCM-SSD scores were evaluated separately in 31 SRs, 26 SRs, 17 SRs, and 6 SRs. There were significant differences in the effect of nondrug paratherapy for lowering the SBP $(n=28,90.3 \%)[44,53,59,63,66,71,73,74,87,91$, $105,106,113,140-153]$, lowering DBP $(n=22,84.6 \%)$ $[44,53,59,63,66,71,73,74,105,140-151]$, improving the total effective rate $(n=16,94.1 \%)[71,90,91,113,140,142$, $143,145,147,148,150,152,154-157]$, and lowering the TCM-SSD scores $(n=6,100.0 \%)[70,71,105,152,156]$.

3.10.4. Nondrug Therapy versus Antihypertensive Drugs. Of the 139 SRs, 24 SRs (17.3\%) involved nondrug therapy. The outcome measures SBP, DBP, total effective rate, and TCM-SSD scores were evaluated in 14 SRs, 12 SRs, 8 SRs, and 6 SRs, respectively. There were significant differences in the effect of nondrug therapy for lowering the SBP $(n=6$, 
TABLE 2: Distribution of research on prevention and treatment of hypertension and the complications by TCM.

\begin{tabular}{lcr}
\hline Complication & Number of research articles $(n(\%))$ & 26.9 \\
\hline Cerebral haemorrhage & 693 & 15.4 \\
Kidney damage & 397 & 14.7 \\
Diabetes mellitus/abnormal glucose metabolism & 378 & 6.7 \\
Left ventricular dysfunction & 173 & 5.1 \\
Hyperlipidaemia & 132 & 4.9 \\
Sleep disorder & 125 & 4.4 \\
Angina & 113 & 4.2 \\
Atherosclerosis & 107 & 4.1 \\
Anxiety and depression & 105 & \\
Cerebral infarction & 76 & \\
Disease of the eyes & 45 & 1.9 \\
Arrhythmia & 40 & 1.6 \\
Hyperuricemia & 27 & 1.1 \\
Metabolic syndrome & 22 & 0.9 \\
Ventricular dysfunction & 22 & 0.3 \\
Hyperviscositemia & 7 & 0.1 \\
Others & 3 & 5.3 \\
\hline
\end{tabular}

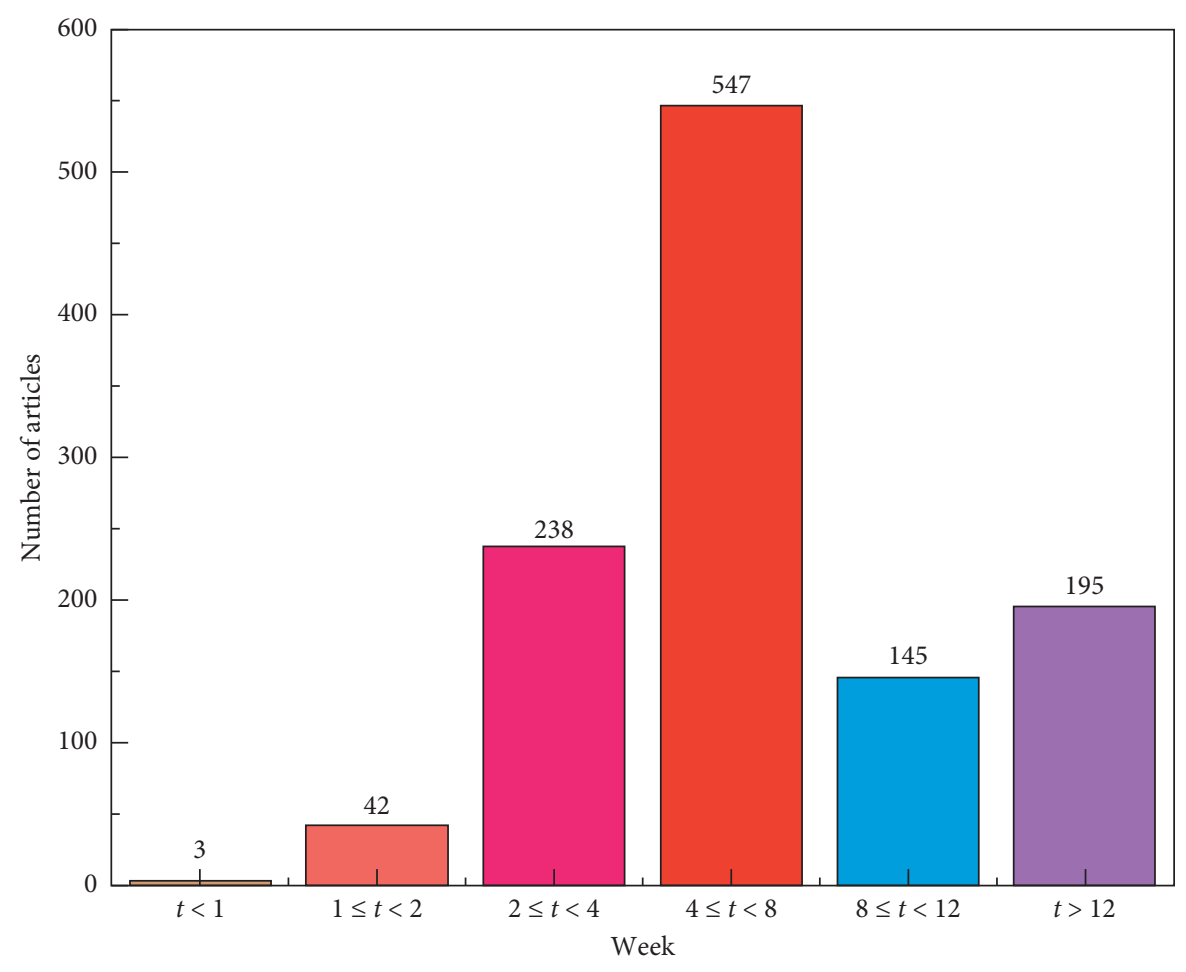

Figure 7: Duration distribution of TCM intervention in hypertension.

$42.8 \%)[44,53,91,105,146,151]$, lowering DBP $(n=6$, $50.0 \%)[44,53,105,113,141,146]$, improving the total effective rate $(n=4,50.0 \%)[91,158-160]$, and lowering the TCM-SSD scores $(n=5,83.3 \%)[70,88,105,156,160]$.

3.11. Potentially Promising Effects in High-Quality (AMSTAR 29) Literature. A total of 10 high-quality studies were retrieved, where qigong (20 RCTs), zhengan xifeng decoction (6 RCTs), Liuwei Dihuang pill (6 RCTs), and CHM (24 RCTs) were considered to have evidence of potential positive effects. Xuefu zhuyu decoction (15 RCTs) was considered a positive effect; i.e., we are confident in estimating the research results. The SRs of acupuncture (22 RCTs), shenqi pill (4 RCTs), jianling decoction (10 RCTs), tongxinluo capsule (25 RCTs), and CHM (5 RCTs) had unclear evidence.

\subsection{Blood Pressure}

3.12.1. CHM versus Antihypertensive Drugs. Zhengan xifeng decoction showed a significant difference in the SBP and DBP control $(P<0.05 ; 4$ RCTs) compared to antihypertensive drugs [7]. In contrast, one SR showed no significant differences [43]. 


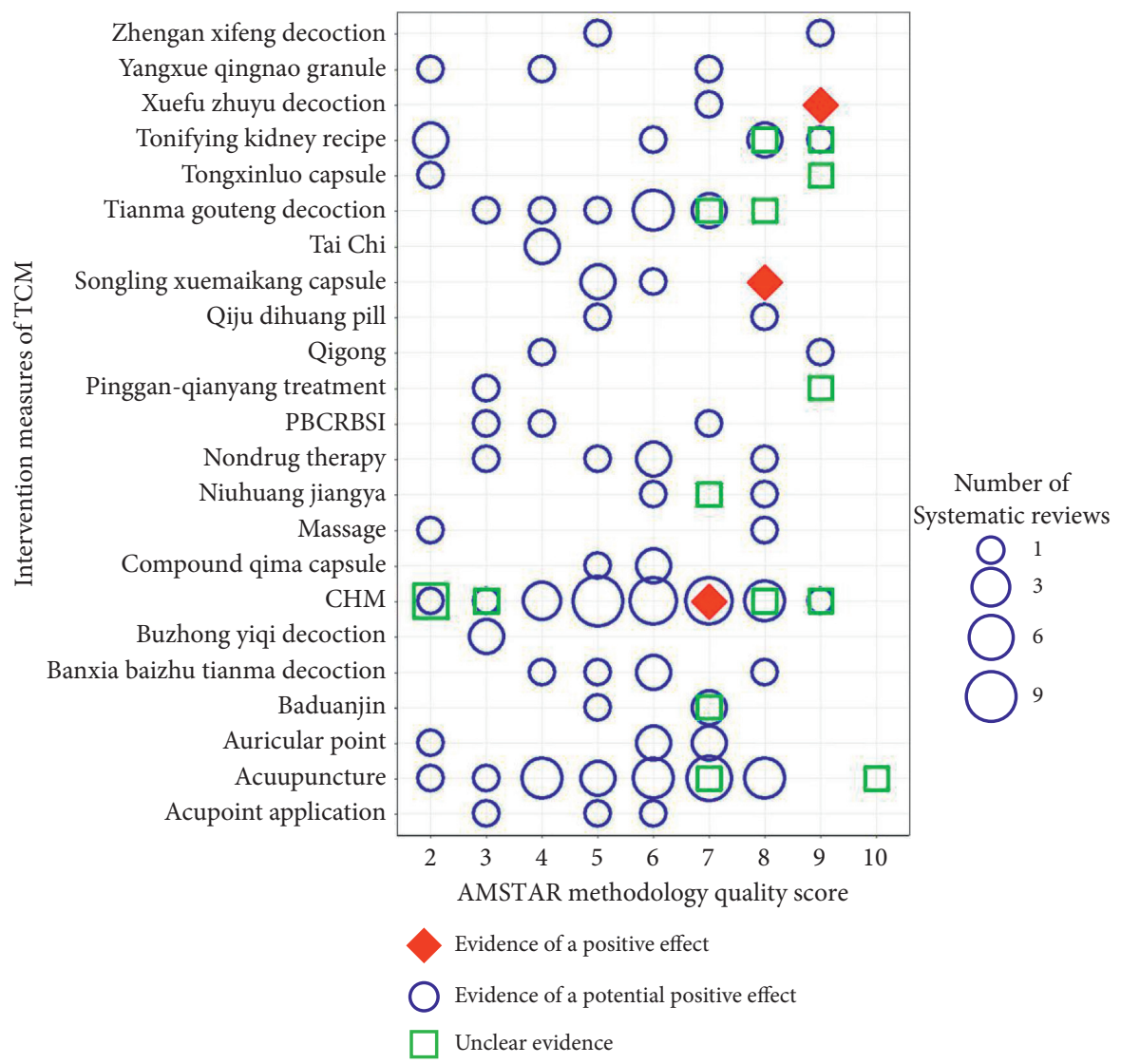

FIGURE 8: Evidence distribution diagram of systematic reviews. The plot depicts the estimated number of SRs (size of the bubble), the clinical efficacy of SRs (shape and colour of the bubble), the AMSTAR scores ( $x$ axis), and the types of TCM intervention ( $y$ axis). Green squares indicate unclear evidence of SRs, blue bubbles indicate potential evidence of SRs, and red rhombus indicate positive evidence of SRs. PBCRBSI: promoting blood circulation and removing blood stasis injection.

3.12.2. CHM plus Antihypertensive Drugs versus Antihypertensive Drugs. The pooled results of the largest review (24 RCTs, 4502 participants) showed a high number of participants with reduced blood pressure (relative risk (RR) 1.28 ; 95\% confidence interval (CI) $1.21,1.36, P<0.001 ; 8$ RCTs (RR: $1.12 ; 95 \%$ CI 1.06, 1.39, $P<0.001 ; 5$ RCTs)). However, the authors cautioned evidence of a potentially positive effect due to the poor quality of the included RCTs [41]. Fifteen studies reported significant effects of xuefu zhuyu decoction combined with antihypertensive drugs (15 RCTs, 1364 participants) for lowering the blood pressure compared to the control group $(P<0.05)$. The author suggested that xuefu zhuyu decoction for hypertension should be prioritised for future preclinical and clinical studies [6]. The Liuwei Dihuag pill (6 RCTs, 555 participants) and jian ling decoction combined with antihypertensive drugs were more effective in controlling the blood pressure $[43,46]$. In contrast, 2 SRs showed no significant difference $[42,47]$.

3.12.3. Nondrug Therapy plus Antihypertensive Drugs versus Antihypertensive Drugs. Qigong plus antihypertensive drugs significantly lowered both the SBP (WMD = $-11.99 \mathrm{mmHg}$; 95\% CI $-15.59,-8.39, P<0.00001)$ and DBP
$(\mathrm{WMD}=-5.28 \mathrm{mmHg} ; 95 \% \mathrm{CI}-8.13,-2.42, P=0.0003 ; 5$ RCTs) compared to the antihypertensive drugs alone. Compared to no intervention, qigong significantly reduced SBP and DBP $(P<0.05)$ [44]. One Cochrane review concluded that the clinical evidence for short-term and sustained BP-lowering effect by acupuncture was unclear (quality $=10)[40]$.

\subsection{TCM-SSD Scores}

3.13.1. CHM versus Antihypertensive Drugs. One SR showed a significant effect of CHM for lowering the TCM-SSD scores compared to the antihypertensive drugs [7].

3.13.2. CHM plus Antihypertensive Drugs versus Antihypertensive Drugs. Two SRs showed a significant effect of $\mathrm{CHM}$ combined with antihypertensive drugs for lowering the TCM-SSD scores compared to the antihypertensive drugs $[41,46]$.

3.14. Adverse Events. Of the 139 SRs, there was an outcome measure of adverse effects in 77 SRs, which included gastrointestinal reaction, dizziness, headache, cough, and nausea $[41,49]$. In summary, all of these SRs indicated that the side effects in the TCM adjuvant therapy group were 
generally less than or lighter than those in the Western medicine group.

\subsubsection{Guidelines, Consensus, and Clinical Pathway Studies.} A total of 16 papers were retrieved on the treatment of hypertension guidelines, consensus, and clinical pathway of TCM research, including the TCM treatment for hypertension and its complications and consensus $(n=10)$, consensus recommendation on the application for CPM of hypertension $(n=1)$ [161], the optimisation path of TCM clinical program $(n=4)$, and the nursing clinical path $(n=1)$ [162]. In 2019, more than $70 \%$ of the experts recommended 6 types of CPM: the Tianma Gouteng decoction, qiju dihuang capsule, jinguishenqi pill, gingko leaf tablets, niuhuang jiangya pill, and banxia tianma pill to help non-TCM practitioners to select appropriate CPM according to the TCM symptoms. In addition, multicentre RWS found that the 7 common syndromes under the TCM diagnosis and treatment guidelines for hypertension, including liver fire flaming upward syndrome, yin deficiency and yang hyperactive syndrome, blood stasis and internal obstruction, phlegm and dampness, deficiency of qi and blood, deficiency of kidney essence, and chong and ren imbalance, only accounted for $58.38 \%$ of the common syndromes. Further, adding the phlegm and blood stasis mutual settlement syndrome is recommended and so it cancels the chong and ren imbalance [163]. Guidelines and path research guide the treatment of EH in TCM and also guide the treatment of complications such as acute cerebral haemorrhage and depression.

\section{Discussion}

In this study, an evidence map was used to systematically sort the literature on hypertension in the past 20 years. Compared to the previous evidence mapping studies that only included RCTs or SRs [11-14], the current study mainly focussed on the diversified research types (observational studies, interventional studies, secondary studies, and RWS), intervention measures (CHM and nondrug therapy), and the analysis contents (TCM prevention and treatment schemes, intervention time, study outcomes, adverse reactions, etc.) has been expanded to provide a comprehensive description of the clinical problem. It shows the volume and field of available research and highlights areas where published meta-analysis has reported positive results and identified gaps in evidence.

4.1. Advantages of TCM in the Prevention and Treatment of Hypertension. For hypertension prevention and treatment by TCM, the key areas to target are lowering BP, lowering the TCM-SSD scores, improving the clinical symptoms, and protecting the target organs. The adverse events in the TCM paratherapy group were generally less than those in the control group. A total of 120 SRs found that CHM and nondrug therapy had potential active effects for the treatment of hypertension, 16 SRs showed unclear evidence, and 3 SRs showed active effects. Regarding complications, damage to the heart, brain, and kidney target organs accounted for more than $50 \%$ of the studies, and TCM had a good effect on the dissipation of the hypertensive cerebral haematoma, stroke score, proteinuria, and left ventricular hypertrophy. Meanwhile, the evaluation of TCM clinical programs showed that TCM combined with Western medicine can enhance clinical effectiveness and reduce adverse events. Regarding clinical symptoms, it had an improved effect on the main symptoms of vertigo, headache, and systemic symptoms. Based on the study of guidelines and pathways, TCM syndromes and CPM (tianma gouteng decoction qiju dihuang capsule, jingui shenqi pill, gingko leaf tablets, niuhuang jiangya pill, and banxia tianma pill) have been put forward for clinical application.

4.2. Future Focus on TCM Prevention and Treatment of Hypertension. TCM intervention for prehypertension is still insufficient. At present, only 3 SRs have been published, including nondrug therapy (17 RCTs, quality=6) [160], CHM (8 RCTs, quality=5) [137], and CHM (5 RCTs, quality $=8$ ) [51]. In the future, greater focus should be placed on improving prevention and treatment during early hypertension, including prehypertension, grade 1 hypertension, and youth hypertension, and additional research should be carried out on specific clinical indicators and mechanisms. It is also important to investigate in emotion, obesity, and other hypertension risk factors by $\mathrm{CHM}$ and nondrug therapy.

4.3. Limitations and Implications. In general, a summary of the findings of included SRs and clinical studies showed that TCM paratherapy for EH has better efficacy and safety than the control group. The research evidence on the risk factors, quality of life, emotional and psychological, early intervention, duration of intervention, and adverse events is weak. However, there are several limitations to the present study. First, the evidence map provides only a broad overview of the research areas and cannot provide definitive answers regarding the effectiveness of an intervention. The specific control of clinical indicators requires more detailed and targeted research. Second, the evidence map did not establish the reporting guidelines and did not avoid overlap between the included studies across reviews. Third, the quality of the methodology of most SRs was low (25.2\%) to moderate (67.6\%), which directly influences the reliability of the results. Fourth, literature types, heterogeneity, and complex intervention measures in the included studies only elucidate the efficacy and safety at a macroscopic level.

The improvements for further evidence map are as follows $[164,165]$. In terms of data sources, a complementary search of the clinical registration platform and references should be additionally conducted. Regarding content extraction, one should further focus on the retrieval according to the priority areas to further improve accuracy. To avoid unrecognised individual literature due to a large number of retrieved literature and the problem of splitting the same research results, topic selection of TCM literature should focus on specific clinical problems, avoid extensive titles, and prevent the result from being too complex for an explanation. Finally, one should review the evidence base with standard evidence synthesis methods (i.e., systematic 
review), improve the methodological quality of SRs themselves, and encourage prospective registration of SRs.

\section{Conclusion}

The conclusion of the SRs and primary studies highlight TCM's advantages as adjunctive therapy for improving hypertension. Similarly, the development trend of CHM and nondrug therapy for the prevention and treatment of hypertension is relatively good, which reflects the diverse TCM prevention and treatment measures for hypertension. However, clinical research evidence needs to be treated with caution because of methodological flaws. In the future, studies with larger sample sizes, standardisation, and higher quality are required to provide further scientific evidence for TCM in treating hypertension.

\section{Data Availability}

The datasets used during the current study are available from the corresponding author upon reasonable request.

\section{Conflicts of Interest}

The authors declare that there are no conflicts of interest regarding the publication of this paper.

\section{Authors' Contributions}

Yue Liu and Fengqin Xu conceived the idea, designed the study, and interpreted the data together and are the cocorresponding authors. Yan Zhang, Biqing Wang, Chunxiao Ju, and Lu Liu conducted the literature searches, evaluated the risk of bias of each study, and wrote the manuscript together. Jun Mei and Ying Zhu helped to revise the manuscript.

\section{Acknowledgments}

This work was supported by the Fundamental Research Funds for the Central Public Welfare Research Institutes (ZZ13-024-4), grants of National Key R\&D Program of China (2017YFC1700301), and Qihuang Scholar of "Millions of Talents Project” (Qihuang Project).

\section{References}

[1] M. Ezzati, A. D. Lopez, A. Rodgers, S. Vander Hoorn, and C. J. Murray, "Selected major risk factors and global and regional burden of disease," The Lancet, vol. 360, no. 9343, pp. 1347-1360, 2002.

[2] P. M. Kearney, M. Whelton, K. Reynolds, P. Muntner, P. K. Whelton, and J. He, "Global burden of hypertension: analysis of worldwide data," The Lancet, vol. 365, no. 9455, pp. 217-223, 2005.

[3] N. Chung, S. Baek, M.-F. Chen et al., "Expert recommendations on the challenges of hypertension in Asia," International Journal of Clinical Practice, vol. 62, no. 9, pp. 1306-1312, 2008.

[4] W. Li, H. Gu, K. K. Teo et al., "Hypertension prevalence, awareness, treatment, and control in 115 rural and urban communities involving 47000 people from China," Journal of Hypertension, vol. 34, no. 1, pp. 39-46, 2016.
[5] F. Guo, H. Di, W. Zhang, and R. G. Walton, "Trends in prevalence, awareness, management, and control of hypertension among United States adults, 1999 to 2010," Journal of the American College of Cardiology, vol. 60, no. 7, pp. 599606, 2012.

[6] P. Wang, X. Xiong, and S. Li, "Efficacy and safety of a traditional Chinese herbal formula xuefu zhuyu decoction for hypertension," Medicine, vol. 94, no. 42, Article ID e1850, 2015.

[7] X. Xiong, X. Yang, B. Feng et al., "Zhen Gan Xi Feng decoction, a traditional Chinese herbal formula, for the treatment of essential hypertension: a systematic review of randomized controlled trials," Evidence-based Complementary and Alternative Medicine, vol. 2013, Article ID 982380, 9 pages, 2013.

[8] I. M. Miake Lye, S. Hempel, R. Shanman, and P. G. Shekelle, "What is an evidence map? A systematic review of published evidence maps and their definitions, methods, and products," Systematic Reviews, vol. 5, no. 1, 2016.

[9] B. Snilstveit, M. Vojtkova, A. Bhavsar, J. Stevenson, and M. Gaarder, "Evidence \& gap maps: a tool for promoting evidence informed policy and strategic research agendas," Journal of Clinical Epidemiology, vol. 79, pp. 120-129, 2016.

[10] M. M. Anaya, J. V. Franco Ariel, M. Ballesteros, I. Solà, G. U. Cuchí, and X. Bonfill Cosp, "Evidence mapping and quality assessment of systematic reviews on therapeutic interventions for oral cancer," Cancer Management and Research, vol. 11, pp. 117-130, 2019.

[11] M. R. Solloway, S. L. Taylor, P. G. Shekelle et al., "An evidence map of the effect of Tai Chi on health outcomes," Systematic Reviews, vol. 5, no. 1, 126 pages, 2016.

[12] I. M. Miake-Lye, S. Mak, J. Lee et al., "Massage for pain: an evidence map," The Journal of Alternative and Complementary Medicine, vol. 25, no. 5, pp. 475-502, 2019.

[13] S. Luger, S. L. Taylor, M. R. Solloway et al., "Evidence map of acupuncture," Department of Veterans Affairs, Washington DC, USA, 2014.

[14] H. Wei, Y. Xiao, Y. Tong et al., "Therapeutic effect of angelica and its compound formulas for hypertension and the complications: evidence mapping," Phytomedicine, vol. 59, Article ID 152767, 2019.

[15] L. Monasta, G. D. Batty, A. Cattaneo et al., "Early-life determinants of overweight and obesity: a review of systematic reviews," Obesity Reviews, vol. 11, no. 10, pp. 695-708, 2010.

[16] M. W. M. Lutje, M. Smeulers, H. Vermeulen, and L. W. Peute, "Effects of clinical decision-support systems on practitioner performance and patient outcomes: a synthesis of high-quality systematic review findings," Journal of the American Medical Informatics Association, vol. 18, no. 3, pp. 327-334, 2011.

[17] D. Y. Peng, "The effect of traditional Chinese medicine on the prevention and treatment of hypertension in community management," Nei Mongol Journal of Traditional Chinese Medicine, vol. 35, no. 14, p. 165, 2016.

[18] P. R. Yang, W. T. Shih, Y. H. Chu, P. C. Chen, and C. Y. Wu, "Frequency and co-prescription pattern of Chinese herbal products for hypertension in Taiwan: a cohort study," BMC Complementary and Alternative Medicine, vol. 15, p. 163, 2015.

[19] J. H. Ma, Z. F. Wang, Y. M. Xie et al., "Study on medical pattern of traditional Chinese medicine and western medicine diagnosia and treatment of hypertension patients in 30034 cases in real world," China Journal of Chinese Materia Medica, vol. 39, no. 18, pp. 3435-3441, 2014. 
[20] M. H. Chen and S. W. Xie, "Investigation and analysis of clinical related factors and TCM syndrome type characteristics in 81 young patients with hypertension," Fujian Journal of Traditional Chinese Medicine, vol. 41, no. 1, pp. 7-8, 2010.

[21] Y. Wu and J. B. Zhong, "Clinical characteristics and efficacy of 105 young patients with essential hypertension," Beijing Journal of Traditional Chinese Medicine, vol. 36, no. 3, pp. 213-216, 2017.

[22] S. S. Ye, "Study on the correlation between heart rate variability, left ventricular mass index and traditional chinese medicine syndrome," M. S. thesis, Guangxi University of Chinese Medicine, Nanning, China, 2018.

[23] M. H. Chen, "Analysis of clinical related factors and syndrome characteristics of 81 young patients with hypertension," in Proceedings of the 11th Annual Conference of Heart Disease Branch of Chinese Society of Traditional Chinese Medicine, Hangzhou, Zhejiang, China, 2009.

[24] Z. Zhao, "The study of the relationship between hypertension TCM syndrome and duration, classification and risk factors of hypertension," M.S. thesis, Guangzhou University of Chinese Medicine, Guangzhou, China, 2015.

[25] M. Pang, Y. Zhang, and C. Wang, "Study on the correlation between the four diagnosis information of traditional Chinese medicine and syndrome factors in 150 patients with hypertension," Liaoning Journal of Traditional Chinese Medicine, vol. 39, no. 4, pp. 606-608, 2012.

[26] L. An, "Study on features of tcm syndromes and target orgens disfunction of the 3 grade hypertension and related factors," D.S. thesis, Shandong University of Traditional Chinese Medicine, Jinan, China, 2012.

[27] C. F. Shen, "Clinical research on TCM syndrome factor in hypertension of rank 3," Nanjing University of Chinese Medicine, M.S. Thesis, 2007.

[28] Y. Tang, "Clinical research for the dampness constitution hypertensive patients' degree of atherosclerosis and related risk factors," Heilongjiang University of Chinese Medicine, M.S. thesis, 2013.

[29] B. H. Zhang, S. Q. Gao, and D. X. Fu, "Analysis of utilization of antihypertensive Chinese patent medicine in Beijing hospital of Ministry of health during 2008-2011," Evaluation and Analysis of Drug-Use in Hospitals of China, vol. 13, no. 2, pp. 133-136, 2013.

[30] Z. D. Zou, N. Liu, P. Guo et al., "Analysis on clinical trealment in hypertension by traditional Chinese medicine for 10 years in Beijing," China Journal of Chinese Materia Medicine, vol. 32, no. 15, pp. 1569-1572, 2007.

[31] F. C. Si and S. X. Li, "Analysis on Chinese medicine medication of essential hypertension in recent 30 years," Journal of Medical Research, vol. 39, no. 3, pp. 46-49, 2010.

[32] L. J. Gao, W. F. Cui, S. F. Wang et al., "Effect of traditional Chinese medicine jiangyabao on arteriosclerosis in middleaged and elderly patients with essential hypertension," Chinese Journal of Gerontology, vol. 38, no. 15, pp. 35873589, 2018.

[33] W. F. Cui, X. H. Fan, S. F. Wang et al., "Effect of long-term use of jiangyabao series of Chinese patent medicines on the outcome of hypertension:a cohort study," Chinese General Practice, vol. 22, no. 1, pp. 101-105, 2019.

[34] W. F. Cui, L. K. Wang, Y. Y. Pan et al., "The effect of prescription of jiangyabao series on cardiovascular and cerebrovascular risk in the treatment of essential hypertension," Chinese Journal of Gerontology, vol. 38, no. 17, pp. 4097-4099, 2018.
[35] M. S. Lee, J.-I. Kim, and E. Ernst, "Is cupping an effective treatment? An overview of systematic reviews," Journal of Acupuncture and Meridian Studies, vol. 4, no. 1, pp. 1-4, 2011.

[36] J. Wang and X. Xiong, "Outcome measures of Chinese herbal medicine for hypertension: an overview of systematic reviews," Evidence-based Complementary and Alternative Medicine, vol. 2012, Article ID 697237, 7 pages, 2012.

[37] Y. Wang, Y. F. Liu, Z. Wang et al., "Traditional Chinese medicine treatment for essential hypertension from 2015 to 2019: an overview of systematic reviews," Chinese Journal of Evidence-Based Medicine, vol. 19, no. 12, pp. 1460-1469, 2019.

[38] Y. T. Yang, L. Ma, X. X. Yang et al., "Traditional Chinese medicine for essential hypertension: an overview of systematic reviews," Chinese Journal of Evidence-Based Medicine, vol. 14, no. 9, pp. 1070-1076, 2014.

[39] X. D. Tan, Y. J. Pan, W. B. Jiang et al., "Acupuncture therapy for essential hypertension: an overview of systematic reviews," Chinese Journal of Evidence-Based Medicine, vol. 10, no. 7, pp. 794-799, 2018.

[40] J. Yang, J. Chen, M. Yang et al., "Acupuncture for hypertension," Cochrane Database of Systematic Reviews, vol. 2018, no. 11, Article ID D8821, 2018.

[41] J. Zhao, "Preliminary clinical observation of a randomized, controlled, double-blind trial of songling xuemakang capsule in the treatment of essential hypertension (grade 1)," M. S. Thesis, Beijing University of Chinese Medicine, Beijing, China, 2017.

[42] X. Xiong, X. Li, Y. Zhang, and J. Wang, "Chinese herbal medicine for resistant hypertension: a systematic review," BMJ Open, vol. 5, no. 1, Article ID e5355, 2015.

[43] X. Xiong, P. Wang, X. Li, and Y. Zhang, "The effect of Chinese herbal medicine jian ling decoction for the treatment of essential hypertension: a systematic review," BMJ Open, vol. 5, no. 2, Article ID e6502, 2015

[44] X. Xiong, P. Wang, X. Li, and Y. Zhang, "Qigong for hypertension a systematic review," Medicine, vol. 94, no. 1, Article ID e3521, 2015.

[45] J. Wang, X. Xiong, and W. Liu, "Chinese patent medicine tongxinluo capsule for hypertension: a systematic review of randomised controlled trials," Evidence-based Complementary and Alternative Medicine, vol. 2014, Article ID 187979, 14 pages, 2014.

[46] J. Wang, K. Yao, X. Yang et al., "Chinese patent medicine Liu Wei di Huang Wan combined with antihypertensive drugs, a new integrative medicine therapy, for the treatment of essential hypertension: a systematic review of randomized controlled trials," Evidence-based Complementary and Alternative Medicine, vol. 2012, Article ID 714805, 7 pages, 2012.

[47] X. Xiong, P. Wang, X. Li, and Y. Zhang, "Shenqi pill, a traditional Chinese herbal formula, for the treatment of hypertension: a systematic review," Complementary Therapies in Medicine, vol. 23, no. 3, pp. 484-493, 2015.

[48] X. Xiong, P. Wang, and S. Li, "Meta-analysis of the effectiveness of traditional Chinese herbal formula Zhen $\mathrm{Wu}$ decoction for the treatment of hypertension," BMJ Open, vol. 5, no. 12, Article ID e7291, 2015.

[49] J. Wang, B. Feng, X. Yang et al., "Tianma Gouteng Yin as adjunctive treatment for essential hypertension: a systematic review of randomized controlled trials," Evidence-based Complementary and Alternative Medicine, vol. 2013, Article ID 706125, 18 pages, 2013. 
[50] D.-Z. Li, Y. Zhou, Y.-N. Yang et al., "Acupuncture for essential hypertension: a meta-analysis of randomized shamcontrolled clinical trials," Evidence-based Complementary and Alternative Medicine, vol. 2014, Article ID 279478, 7 pages, 2014.

[51] J. Wang, B. Feng, X. Yang, W. Liu, and X. Xiong, "Chinese herbal medicine for the treatment of prehypertension," Evidence-based Complementary and Alternative Medicine, vol. 2013, Article ID 493521, 9 pages, 2013.

[52] H. S. Ding and X. F. Zhou, "Meta-analysis of the antihypertensive effect of 442 cases of TCM decoction," Practical Journal of Clinical Medicine, vol. 9, no. 4, pp. 192-194, 2012.

[53] J. Qian and Z. Z. Sun, "Systematic evaluation of ba duan jin in the adjuvant treatment of hypertension," Chinese Journal of Ethnomedicine and Ethnopharmacy, vol. 27, no. 8, pp. 52-57, 2018.

[54] H. Wang, H. C. Shang, J. H. Zhang et al., "Niuhuang jiangya preparation for treatment of essential hypertension: a systematic review," Liaoning Journal of Traditional Chinese Medicine, vol. 5, pp. 649-652, 2008.

[55] Z. Q. Zhi, "Meta-analysis of the clinical efficacy of traditional Chinese medicine in the treatment of hypertension," Chinese Medicine Modern Distance Education of China, vol. 15, no. 7, pp. 57-58, 2017.

[56] Y. P. Wu and J. G. Zhang, "Meta-analysis of the antihypertensive effect of traditional Chinese medicine on essential hypertension," Lishizhen Medicine and Materia Medica Research, vol. 23, no. 9, pp. 2360-2361, 2012.

[57] Y. Ren, A. H. Ou, and X. Z. Lin, "Meta-analysis of a randomized controlled trial of traditional Chinese medicine in the treatment of essential hypertension," Shaanxi Journal of Traditional Chinese Medicine, vol. 7, pp. 794-796, 2006.

[58] J. Wang, "Systematic evaluation of gastrodia elata hooteng decoction in the treatment of hypertension," M.S. thesis, Shaanxi University of Traditional Chinese Medicine, Xianyang, China, 2013.

[59] J. Wang, X. Xiong, and W. Liu, "Acupuncture for essential hypertension," International Journal of Cardiology, vol. 169, no. 5, pp. 317-326, 2013.

[60] X. Tan, Y. Pan, W. Su et al., "Acupuncture therapy for essential hypertension: a network meta-analysis," Annals of Translational Medicine, vol. 7, no. 12, p. 266, 2019.

[61] X. Gong, X. Yang, W. Liu et al., "Banxia baizhu tianma decoction for essential hypertension: a systematic review of randomized controlled trials," Evidence-based Complementary and Alternative Medicine, vol. 2012, p. 10, Article ID 271462, 2012.

[62] J. Wang, X. Xiong, G. Yang et al., "Chinese herbal medicine Qi Ju di Huang Wan for the treatment of essential hypertension: a systematic review of randomized controlled trials," Evidence-based Complementary and Alternative Medicine, vol. 2013, p. 10, Article ID 262685, 2013.

[63] X. Yang, H. Zhao, and J. Wang, "Chinese massage (Tuina) for the treatment of essential hypertension: a systematic review and meta-analysis," Complementary Therapies in Medicine, vol. 22, no. 3, pp. 541-548, 2014.

[64] X. Xiong, P. Wang, L. Duan et al., "Efficacy and safety of Chinese herbal medicine Xiao Yao San in hypertension: a systematic review and meta-analysis," Phytomedicine, vol. 61, Article ID 152849, 2019.

[65] H.-C. Liu, J.-Q. Ju, Y.-L. Li et al., "Efficacy of Chinese herbal medicine on health-related quality of life (SF-36) in hypertensive patients: a systematic review and meta-analysis of randomized controlled trials," Complementary Therapies in Medicine, vol. 23, no. 3, pp. 494-504, 2015.

[66] X. Ma, H. Hu, J. Li et al., "Is acupuncture effective for hypertension? A systematic review and meta-analysis," PLoS One, vol. 10, Article ID e01270197, 2015.

[67] Y. Cao, L.-t. Liu, and M. Wu, "Is Chinese herbal medicine effective for elderly isolated systolic hypertension? A systematic review and meta-analysis," Chinese Journal of Integrative Medicine, vol. 23, no. 4, pp. 298-305, 2017.

[68] J. Wang, X. Yang, B. Feng et al., "Is yangxue qingnao granule combined with antihypertensive drugs, a new integrative medicine therapy, more effective than antihypertensive therapy alone in treating essential hypertension?" Evidencebased Complementary and Alternative Medicine, vol. 2013, Article ID 540613, 8 pages, 2013.

[69] J. Lan, Y. Zhao, F. Dong et al., "Meta-analysis of the effect and safety of berberine in the treatment of type 2 diabetes mellitus, hyperlipemia and hypertension," Journal of Ethnopharmacology, vol. 161, pp. 69-81, 2015.

[70] X.-J. Yan, P.-Q. Wang, and S.-J. Li, "Blood-letting therapy for hypertension: a systematic review and meta-analysis of randomized controlled trials," Chinese Journal of Integrative Medicine, vol. 25, no. 2, pp. 139-146, 2019.

[71] N. Hou, Y. Huang, R. H. Chen, and Z. X. Zhang, "Meta analysis of the clinical efficacy of moxibustion in the treatment of essential hypertension," Shi Zhen Guo Yi Guo Yao, vol. 30, no. 10, pp. 2519-2524, 2019.

[72] X. Y. Chen, J. Y. Zhao, G. Q. Zhang et al., "Meta analysis of the effect of baduanjin on blood pressure in patients with hypertension," China Medical Herald, vol. 15, no. 24, pp. 137-140, 2018.

[73] J. H. Lin and R. Li, "Systematic evaluation and meta-analysis of the efficacy of badan jinlian in the treatment of essential hypertension with conventional regimens," Journal of Guangzhou University of Traditional Chinese Medicine, vol. 34, no. 5, pp. 774-780, 2017.

[74] Z. S. Chen, L. W. Zheng, C. C. Yang et al., "Effects of baduanjin exercise on patients with hypertension: a metaanalysis," Nursing Journal of Chinese People's Liberation Army, vol. 35, no. 10, pp. 1-8, 2018.

[75] M. Zhu and Y. L. Li, "Systematic evaluation of the effect of pinellia atractylodes rhizome gastrodia elata decoction and its addition and reduction prescription on serum lipid level in patients with hypertension associated with hyperlipidemia," Journal of Changchun University of Traditional Chinese Medicine, vol. 29, no. 6, pp. 980-982, 2013.

[76] M. Zhu, J. Q. Ju, and Y. L. Li, "Systematic review of randomized controlled trials of banxia baizhu tianma decoction on essential hypertension with phlegm-dampness pattern," Journal of Shandong University of Traditional Chinese Medicine, vol. 38, no. 2, pp. 105-108, 2014.

[77] J. Juan, "Clinical efficacy evaluation of acupoint selection based on syndrome differentiation and meridians in the treatment of essential hypertension," D. S. thesis, Chengdu University of TCM, Chengdu, China, 2015.

[78] J. X. Zou, W. P. Wang, and L. T. Lou, "An meta analysis of the treatment of hypertension by method of invigorating kidney and promoting blood circulation," Henan Traditional Chinese Medicine, vol. 39, no. 11, pp. 1737-1743, 2019.

[79] X. Z. Lu, "Systematic evaluation of kidney-tonifying prescription in the treatment of essential hypertension," M.S. thesis, Shandong University of Chinese Medicine, Jinan, China, 2017. 
[80] J. H. Liang, M. Chen, Y. H. Cai et al., "The efficacy of bushen yixin tablets with western medicine for the treatment of hypertension: meta analysis and systematic review," Chinese Journal of Integrative Medicine on Cardio-/Cerebrovascular Disease, vol. 15, no. 19, pp. 2372-2376, 2017.

[81] M. Shi and Y. H. Zhang, "Systematic evaluation of invigorating kidney-qi therapy for deficiency of kidney-qi syndrome in essential hypertension," Shandong Journal of Traditional Chinese Medicine, vol. 31, no. 4, pp. 236-238, 2012.

[82] C. J. Song and X. Zhuang, "Meta-analysis of the antihypertensive effect of buzhong yiqi decoction on qi-deficiency hypertension," Hunan Journal of Traditional Chinese Medicine, vol. 34, no. 2, pp. 118-119, 2018.

[83] X. B. Wang and L. Xiao, "Meta analysis of modified buzhong yiqi decoction for treating hypertension," Shanxi Journal of Traditional Chinese Medicine, vol. 32, no. 9, pp. 46-48, 2016.

[84] L. Liu and Y. L. Li, "Systematic review on treatment of essential hypertension from spleen and kidney deificency," China Journal of Traditional Chinese Medicine and Pharmacy, vol. 26, no. 8, pp. 700-703, 2011.

[85] Q. Wang, "Meta analysis of efficacy and safety of danshen injection in the treatment of hypertensive cerebral hemorrhage," M.S. thesis, Zhejiang University, Hangzhou, China, 2012.

[86] Q. X. Song, D. Z. Cui, and Z. Wang, "Systematic evaluation of the effect of danhong injection on renal function in hypertensive patients with renal damage," Shandong Medical Journal, vol. 57, no. 25, pp. 78-80, 2017.

[87] X. Meng, Q. Zhang, Y. J. Zhang et al., "Meta-analysis of the clinical efficacy of auricular apex bloodletting in the treatment of hypertension," Guiding Journal of Traditional Chinese Medicine and Pharmacy, vol. 25, no. 14, pp. 120-124, 2019.

[88] H. H. Zhang, X. Y. Wang, and J. H. Song, "Meta-analysis of the therapeutic effect of ear buries on primary hypertension nursing," Scientific \& Technical Information of Gansu, vol. 46, no. 2, pp. 89-90, 2017.

[89] H. L. Zhang, Q. L. Chen, M. Zhang et al., "Auricular pressure therapy for mild-to-moderate essential hypertension:a metaanalysis trials," Chinese Journal of Integrative Medicine on Cardio-/Cerebrovascular Disease, vol. 14, no. 17, pp. 19661970, 2016.

[90] Y. N. Shi, H. M. Guan, and Q. H. Sun, "Meta analysis of clinical effect of ear acupoint application adjuvant therapy for hypertension patients," Nursing Research of China, vol. 31, no. 18, pp. 2229-2232, 2017.

[91] Q. Q. Liu, X. C. Liu, J. Liu et al., "Auricular acupuncture for essential hypertension: a systematic review," Asia-Pacific Traditional Medicine, vol. 12, no. 21, pp. 38-43, 2016.

[92] Z. M. Zhao, W. Ren, P. J. Du et al., "Depressor effect of compound qima capsule on hypertension: a meta-analysis," Chinese Manipulation \& Rehabilitation Medicine, vol. 9, no. 24, pp. 47-52, 2018.

[93] Z. M. Zhao, N. N. Xia, and W. Ren, "Meta-analysis of the self-controlled study on intervention of compound qima capsule on blood pressure and blood lipid in patients with hypertension," Clinical Journal of Chinese Medicine, vol. 11, no. 30, pp. 18-24, 2019.

[94] Z. M. Zhao, N. N. Xia, J. P. Du et al., "Safety and TCM curative efficacy of compound qi ma capsule for hypertension: a meta analysis," Traditional Chinese Drug Research and Clinical Pharmacology, vol. 30, no. 5, pp. 614-621, 2019.
[95] D. M. De and X. M. Fang, "Meta-analysis of the integrated treatment of traditional Chinese and western medicine and the therapeutic effect of western medicine on hypertension," Chinese Journal of Integrative Medicine on Cardio-/Cerebrovascular Disease, vol. 11, no. 5, pp. 518-521, 2013.

[96] P. P. Sun and X. Jin, "Meta-analysis of the effect of puerarin on hypertension," Journal of Qiqihar University of Medicine, vol. 38, no. 13, pp. 1492-1497, 2017.

[97] W. Guo, "Auxiliary effect of safflower injection for essential hypertension in China: a systematic review," Journal of Liaoning University of Traditional Chinese Medicine, vol. 15, no. 9, pp. 109-111, 2013.

[98] W. Guo, "Systematic evaluation of radix astragali injection in the treatment of renal damage in essential hypertension," Journal of Shandong University of Traditional Chinese Medicine, vol. 37, no. 5, pp. 375-377, 2013.

[99] Y. J. Zhao, X. H. Su, and Y. Y. Guo, "Systematic evaluation and meta-analysis of the efficacy and safety of the randomized controlled clinical trial of activating blood circulation and removing blood stasis in the treatment of essential hypertension," Chinese Journal of Integrative Medicine on Cardio-/Cerebrovascular Disease, vol. 15, no. 12, pp. 14171427, 2017.

[100] A. Li, "Clinical safety evaluation of representative expectorant containing pinellia chinensis based on meta-analysis," M.S. thesis, Beijing University of Chinese Medicine, Beijing, China, 2019.

[101] H. Yu, "Clinical evaluation of chinese patent medicine in the treatment of essential hypertension based on meta-analysis and data mining," D.S. thesis, China Academy of Chinese Medical Sciences, Beijing, China, 2019.

[102] J. H. Ma, "Based on meta-analysis and clinical study of songling xuemai kang, the therapeutic effect of pinggan method on hypertension was evaluated," D.S. thesis, China Academy of Chinese Medical Sciences, Beijing, China, 2018.

[103] S. S. Zhang, "A meta-analysis of the treatment of hypertension with longdan xiegang decoction based on a randomized controlled trial," M.S. thesis, Shandong University of Traditional Chinese Medicine, Jinan, China, 2016.

[104] Y. W. Song, "A meta-analysis of the treatment of hypertension with bezoar antihypertensive prescription based on a randomized controlled trial," M.S. thesis, Shandong University of Traditional Chinese Medicine, Jinan, China, 2016.

[105] C. Y. Ma, "A meta-analysis of acupuncture therapy for hypertension based on randomized controlled trials," M.S. thesis, Shandong University of Traditional Chinese Medicine, Jinan, China, 2016.

[106] H. Chen, "Establishment of evidence system for acupuncture treatment of essential hypertension based on reticular analysis," D.S. thesis, Nanjing University of Chinese Medicine, Nanjing, China, 2019.

[107] W. F. Cui, B. C. Wang, J. M. Fan et al., "A meta analysis of jiang ya Bao in treatment of essential hypertension," Clinical Journal of Traditional Chinese Medicine, vol. 27, no. 6, pp. 849-850, 2015.

[108] X. Y. Cui, X. Z. Ma, and Q. Y. Qiu, "Systematic evaluation of intervention of jingui shenqi pills in hypertension," Chinese Journal of Information on Traditional Chinese Medicine, vol. 25, no. 7, pp. 87-91, 2018.

[109] D. Y. De, Z. J. Zhi, and D. G. Liu, "Meta analysis of the effect of traditional Chinese medicine nursing intervention in elderly hypertension community," Clinical Journal of Traditional Chinese Medicine, vol. 29, no. 7, pp. 1036-1039, 2017. 
[110] H. Yu, J. Mei, P. Zhang et al., "Systematic evaluation of the efficacy and safety of bezoar antihypertensive Chinese patent medicine in the treatment of essential hypertension," Beijing Journal of Traditional Chinese Medicine, vol. 38, no. 5, pp. 487-491, 2019.

[111] W. J. Xu and Y. L. Li, "Systematic review of clinical evidence about calm the liver and subdue yang therapy on the hypertension disease with syndrome of upper hyperactivity of liver yang," China Journal of Traditional Chinese Medicine and Pharmacy, vol. 27, no. 3, pp. 736-739, 2012.

[112] J. Q. Ju, Y. L. Li, and C. H. Yang, "Systematic review of clinical efficacy and safety of qiju dihuang pills in treating essential hypertension," Journal of Shandong University of Traditional Chinese Medicine, vol. 37, no. 5, pp. 363-367, 2013.

[113] W. Xiao, W. C. Zhang, and X. F. Chen, "Analysis of the effect of qigong exercise in the treatment of hypertension meta," Journal of Jiangxi University of Traditional Chinese Medicine, vol. 27, no. 2, pp. 49-56, 2015.

[114] H. Yu, J. Mei, P. Zhang et al., "Efficacy and safety of qingnao jiangya patent medicine in the treatment of primary hypertension: a systematic review," Chinese Journal of Integrative Medicine on Cardio-Cerebrovascular Disease, vol. 17, no. 7, pp. 961-966, 2019.

[115] X. X. Lang, "Meta analysis of the treatment of hypertension by song ling xue mai kang capsule based on the randomized controlled trial," M.S. thesis, Shandong University of Traditional Chinese Medicine, Jinan, China, 2016.

[116] L. Chen, "Meta analysis of the treatment of by using the randomized controlled trials of the xinmaitong capsule," M.S. thesis, Shandong University of Traditional Chinese Medicine, Jinan, China, 2016.

[117] S. H. Han, "Antihypertensive effect and quality of life evaluation of combination of Chinese and western medicine in the treatment of senile hypertension," M.S. thesis, China Academy of Chinese Medical Sciences, Beijing, China, 2011.

[118] Y. Cao, "Meta-analysis of the efficacy of traditional Chinese medicine in elderly patients with simple systolic hypertension," M.S. thesis, Beijing University of Chinese Medicine, Beijing, China, 2017.

[119] X. Lu and J. C. Jiang, "Meta-analysis of clinical efficacy of Chinese medicine in treatment of insulin resistance (IR) of hypertension," Chinese Archives of Traditional Chinese Medicine, vol. 37, no. 2, pp. 460-465, 2019.

[120] D. N. Dong and C. H. Yang, "Effects of Chinese medicine on elderly isolated systolic hypertension :a meta-analysis," Liaoning Journal of Traditional Chinese Medicine, vol. 39, no. 5, pp. 812-815, 2012.

[121] J. Dai, "Traditional Chinese medicine for essential hypertension: a systematic review," Journal of China Traditional Chinese Medicine Information, vol. 2, no. 36, pp. 22-24, 2010.

[122] W. L. Hu, R. Tang, L. J. Lu et al., "A systematic review on songlingxuemaikang capsule combined ARB for treating hypertension," China Pharmaceuticals, vol. 23, no. 4, pp. 22-25, 2014.

[123] M. Guo, "Meta analysis on the treatment of essential hypertension with rhizoma gastrodia corydalis combined with amlodipine besylate," M.S. thesis, University of Jinan, Jinan, China, 2016.

[124] B. Q. Lu, Y. H. Ge, X. F. Wu et al., "Systematic Review and meta analysis of tian-ma-gou-teng-yin combined with nifedipine in the treatment of essential hypertension," $\mathrm{Nei}$ Mongol Journal of Traditional Chinese Medicine, vol. 35, no. 12, pp. 52-54, 2016.
[125] Q. Y. Zhou, K. Q. Ma, Y. X. Guo et al., "Meta-analysis of tianma gouteng decoction combined with angiotensinconverting enzyme inhibitors for treating primary hypertension," Chinese Journal of Experimental Traditional Medical Formulae, vol. 21, no. 4, 2015.

[126] W. Liu, F. Y. Zhu, R. Yuan et al., "Efficacy and safety of tongxinluo capsule for hypertension: a systematic review," Beijing Journal of Traditional Chinese Medicine, vol. 34, no. 3, pp. 196-201, 2015.

[127] J. Q. Ju, Y. L. Li, and Z. Z. Shen, "Efficacy of zhengan xifeng decoction treating essential hypertension: a systematic review," Journal of Emergency in Traditional Chinese Medicine, vol. 23, no. 6, pp. 1060-1063, 2014.

[128] J. H. Liang, C. G. Chen, and X. T. Jiang, "Efficacy of wenxin granule and metoprolol in the treatment of hypertensive heart disease complicated with ventricular premature meta analysis and trial sequential analysis," Chinese Journal of Integrative Medicine on Cardio-Cerebrovascular Disease, vol. 17, no. 2, pp. 161-169, 2019.

[129] S. H. Luo, L. J. Zhao, Y. Li et al., "Systematic evaluation of the efficacy of TCM syndrome differentiation or combination of western medicine in the treatment of hypertensive left ventricular hypertrophy," Traditional Chinese Medicine Journal, vol. 16, no. 6, 2017.

[130] Y. Q. Zhao, "Meta-analysis of the clinical efficacy of gastrodia gastrodiae hooteng decoction plus or minus in the treatment of hyperactivity of liver yang in patients with hypertension," M. S. thesis, Shaanxi University of Traditional Chinese medicine, Xianyang, China, 2017.

[131] W. Guo, W. Guo, and Y. Liu, "Systematic evaluation of a randomized controlled trial of gastrodia elata hooteng decoction in the treatment of primary hypertension with hyperactivity of liver yang," Shandong Journal of Traditional Chinese Medicine, vol. 32, no. 11, pp. 794-796, 2013.

[132] J. Zhao, J. H. Du, G. L. Wang et al., "Meta-analysis on randomized controlled trials of Chinese herbal medicine treating essential hypertension," China Journal of Traditional Chinese Medicine and Pharmacy, vol. 33, no. 3, pp. 922-926, 2018.

[133] Q. Wang and S. X. Xi, "Meta-analysis on randomized controlled trials of Chinese herbal medicine treating essential hypertension," China Journal of Traditional Chinese Medicine and Pharmacy, vol. 19, no. 9, pp. 345-349, 2013.

[134] X. N. An, "Meta analysis of efficacy and safety of traditional Chinese medicine in the treatment of early and middle stage renal damage in hypertension," M. S. thesis, Beijing University of Chinese Medicine, Beijing, China, 2019.

[135] S. F. Chen, T. Li, M. L. Li et al., "Xuefu Zhuyu recipe for the treatment of essential hypertension: a systematic review and meta-analysis," Tianjin Journal of Traditional Chinese Medicine, vol. 36, no. 9, pp. 882-890, 2019.

[136] J. Chen, Y. Liu, and J. C. Zhang, "Meta analysis of effect of Chinese medicine on inflammatory response in hypertensive patients," Journal of Medical Research, vol. 42, no. 6, pp. 48-51, 2013.

[137] H. J. Liu, Q. Xu, and X. H. Liu, "Effect of Chinese herbal medicine on blood pressure in pre-hypertensive cases: a meta-analysis," World Chinese Medicine, vol. 11, no. 11, pp. 2441-2443, 2016.

[138] Q. Q. Wu, "Meta-analysis of the clinical efficacy of wendan decoction and pinellia atractylodia rhizome decoction in the treatment of hypertension," Traditional Chinese Medicinal Research, vol. 28, no. 8, pp. 72-74, 2015. 
[139] H. S. Ding, "Meta analysis of traditional chinese medicine of lowering blood pressure deficiency," M. S. thesis, North Sichuan Medical College, Nanchong, China, 2011.

[140] W. W. Li, X. Y. Zhang, and S. S. Luo, "Meta-analysis of the clinical efficacy of acupoint application of traditional Chinese medicine in the treatment of hypertension," The Journal of Practical Medicine, vol. 31, no. 19, pp. 3237-3240, 2015.

[141] Q. Tian, "Clinical study on the treatment of primary hypertension with hyperactivity of liver yang by acupuncture at yuanluo and acupoint," D. S. thesis, Guangzhou University of Chinese Medicine, Guangzhou, China, 2014.

[142] T. Zhu and L. Ding, "Meta analysis of acupuncture on fengchi and quchi acupoint in the treatment of primary hypertension," Clinical Journal of Traditional Chinese Medicine, vol. 30, no. 3, pp. 461-465, 2018.

[143] Y. Y. Chen, J. B. Zhai, T. Shi et al., "Meta-analysis of the clinical efficacy of acupuncture at renying point in the treatment of essential hypertension," Journal of New Chinese Medicine, vol. 49, no. 1, pp. 184-188, 2017.

[144] J. P. Zhang, "Study on fcMRI brain function connection in patients with primary hypertension by acupuncture at taixi point," M. S. thesis, Southern Medical University, Guangzhou, China, 2017.

[145] R. Zhao, L. X. Fu, and J. Xiong, "The effect of acupuncture therapy on essential hypertension: a systematic review of long-term effect," Journal of Clinical Acupuncture and Moxibustion, vol. 27, no. 3, pp. 46-51, 2011.

[146] L. L. Zhang, H. Kang, C. Yang et al., "Effect of acupuncture for hypertension and frequency of acupoints," Liaoning Journal of Traditional Chinese Medicine, vol. 40, no. 10, pp. 2115-2119, 2013.

[147] W. Guo, Q. W. Pei, W. L. Dong et al., “A systematic review of the literature on the combination of acupuncture and medicine in the treatment of essential hypertension," Journal of Shandong University of Traditional Chinese Medicine, vol. 37, no. 2, pp. 99-100, 2013.

[148] Q. Zhao, H. S. Li, M. Q. Ji et al., "Systematic review and metaanalysis of efficacy and safety of massage in treatment of essential hypertension," Journal of Traditional Chinese Medicine, vol. 59, no. 18, pp. 1568-1573, 2018.

[149] K. Y. Liu, N. H. Zhu, and J. X. Peng, "Meta-analysis of intervention effect of TCM nursing in elderly hypertension community," Clinical Journal of Traditional Chinese Medicine, vol. 29, no. 9, pp. 1454-1458, 2017.

[150] H. G. Li and X. Z. Wen, "Systematic evaluation of tai chi in the treatment of essential hypertension," Science \& Teconology Echnology of Stationery \& Sporting Goods, vol. 7, pp. 35-37, 2011.

[151] L. Cai and X. Li, "Meta-analysis of curative effect of taichi on primary hypertension," Clinical Journal of Traditional Chinese Medicine, vol. 28, no. 10, pp. 1425-1428, 2016.

[152] Y. J. Zhang, Z. J. Li, and Y. Gao, "Meta-analysis on efficacy of acupuncture and acupuncture combined with medicine in treatment for mild to moderate essential hypertension," Liaoning Journal of Traditional Chinese Medicine, vol. 41, no. 9, pp. 1802-1806, 2014.

[153] Y. X. Qian, "Systematic evaluation of the efficacy and safety of acupuncture in the treatment of essential hypertension," Journal of North Pharmacy, vol. 10, no. 3, pp. 72-74, 2013.

[154] G. Y. Zhang, S. P. Ou, and Q. D. Zhou, "Meta-analysis of clinical effect of acupoint sticking supplementary therapy of fructus evodiae on hypertension," Chinese Journal of Basic Medicine in Traditional Chinese Medicine, vol. 24, no. 12, pp. 1757-1761, 2018.
[155] M. W. Sun, C. Wang, and S. J. Wang, "Meta analysis on yongquan point sticking therapy for high blood pressure," Journal of Liaoning University of Traditional Chinese Medicine, vol. 19, no. 11, pp. 136-138, 2017.

[156] F. S. Liu, C. Q. Guo, and X. F. Jin, "Acupuncture for mild-tomoderate essential hypertension: a meta-analysis of randomized clinical trials," Chinese Journal of Basic Medicine in Traditional Chinese Medicine, vol. 18, no. 4, pp. 421-423, 2012.

[157] X. M. Chen, N. Q. Shao, X. Ma et al., "Meta analysis and evaluation system of non-drug therapy about traditional Chinese medicine in treatment of essential hypertension," Jilin Journal of Traditional Chinese Medicine, vol. 36, no. 9, pp. 887-890, 2016.

[158] S. Han, L. D. Zhang, L. Y. Gu et al., "Meta-analysis of acupuncture and tianma gouteng decoction in treatment of hypertension," Journal of Practical Traditional Chinese Internal Medicine, vol. 33, no. 11, pp. 5-8, 2019.

[159] Z. H. Tang, "Systematic evaluation of acupuncture treatment of essential hypertension," M. S. thesis, Chengdu University of TCM, Chengdu, China, 2011.

[160] Y. R. Ouyang, C. Y. Huang, W. W. Fu et al., "Meta-analysis of external therapy of traditional Chinese medicine for highnormal blood pressure," Journal of Yunnan University of Traditional Chinese Medicine, vol. 42, no. 2, pp. 31-38, 2019.

[161] X. J. Valaskatgis, "Consensus recommendations on the clinical application of chinese patent drugs in hypertension," in Proceedings of 2011 Annual Conference of Heart DiseaseBranch of Chinese Association of Traditional Chinese Medicineand Annual Meeting of Cardiovascular Diseases Committee of Beijing Association of Traditional Chinese Medicine, Beijing, China, pp. 232-237, 2011.

[162] X. F. Yong and X. F. Yong, "Influence of traditional Chinese medicine clinical nursing pathway on blood pressure level and negative emotion of hypertensive patients," Today Nurse, vol. 25, no. 2, pp. 132-135, 2018.

[163] L. Y. Wang, Y. Li, X. J. Han et al., "Applicability analysis of TCM diagnosis and treatment guideline for hypertension in the real world," China Journal of Traditional Chinese Medicine and Pharmacy, vol. 29, no. 10, pp. 3250-3252, 2014.

[164] M. Chen, Y. Xiao, Y. Liu et al., "The quality analysis of literature retrievals of systematic reviews for traditional Chinese medicine," Journal of Evidence-Based Medicine, vol. 8, no. 1, pp. 42-52, 2015.

[165] J. Zhang, Y. Li, B. Zhang et al., "Evidence-based traditional Chinese medicine research: Beijing declaration," Journal of Evidence-Based Medicine, vol. 13, no. 2, pp. 91-92, 2020. 